\title{
Modeling wake effects in large wind farms in complex terrain: the problem, the methods and the issues
}

\author{
E.S. Politis J. Prospathopoulos D. Cabezon K. S. Hansen P. K. Chaviaropoulos and \\ R. J. Barthelmie
}

\begin{abstract}
Computational fluid dynamic (CFD) methods are used in this paper to predict the power production from entire wind farms in complex terrain and to shed some light into the wake flow patterns. Two full three-dimensional Navier-Stokes solvers for incompressible fluid flow, employing $k-\varepsilon$ and $k-\omega$ turbulence closures, are used. The wind turbines are modeled as momentum absorbers by means of their thrust coefficient through the actuator disk approach. Alternative methods for estimating the reference wind speed in the calculation of the thrust are tested. The work presented in this paper is part of the work being undertaken within the UpWind Integrated Project that aims to develop the design tools for next generation of large wind turbines. In this part of UpWind, the performance of wind farm and wake models is being examined in complex terrain environment where there are few pre-existing relevant measurements. The focus of the work being carried out is to evaluate the performance of CFD models in large wind farm applications in complex terrain and to examine the development of the wakes in a complex terrain environment. Copyright $\bigcirc 2011$ John Wiley \& Sons, Ltd.
\end{abstract}

\section{KEYWORDS}

wake effects; wind farms; complex terrain; turbulence models

\section{INTRODUCTION}

The 2020 and 2030 targets set by the European Commission with the aim of combating global warming (according to the European Wind Energy Association, the goal for 2020 is $20 \%$ penetration, which is equivalent to $230 \mathrm{GW}$ of wind energy capacity, including $40 \mathrm{GW}$ offshore, whereas the goal for 2030 is $400 \mathrm{GW}$ ) define a challenging framework for the entire wind energy community to explore areas of high wind potential such as deep offshore and mountainous onshore. Despite the fact that offshore installations are supposed to comprise the major element of wind energy penetration in the coming years, onshore installations will certainly continue to contribute to the overall growth of the wind energy market.

In a wind farm, the interaction of the flow with wind turbines (W/Ts) results in lower power and higher loads at turbines experiencing wakes compared with those experiencing free-stream conditions. As wakes move downstream, they impact with the ground and are subject to downstream and lateral merging with other ones. The size and degradation of the wake depends on many factors such as the ambient wind speed and turbulence, the wake added turbulence, the turbine type, the terrain, the structure of the boundary layer relating to atmospheric stability, and the flow angle and variation with direction. Most wind farm models were developed in the 1980's for small wind farms in flat or moderately complex terrain ${ }^{1-4}$ and do not fully account for effects related to complex terrain.

It is understood that complex terrain has a significant impact on the local wind climate. The wind speed and the wind direction, as well as the turbulence, are all highly influenced by the local terrain topography. Steep mountain ridges are often responsible for severe flow turning and wind rose narrowing, as well as for possible inversion of the wind shear and downstream flow separation. The prediction of power losses and loading due to wakes in complex terrain requires detailed 
understanding of the flow and the interaction of complex atmospheric structures with those generated by W/Ts. Hence, it clearly calls for flow modeling of increased accuracy.

The work presented in this paper is part of the research being undertaken within the UpWind Integrated Project that aims to develop the design tools for the next generation of large W/Ts. In this part of UpWind, the performance of wind farm and wake models is being examined in complex terrain (and offshore) environments where there are few pre-existing measurements of ambient wind and turbulence and even fewer studies of power estimations. The inherent non-linearity of the flow in complex terrain requires modeling that is based on computational fluid dynamics (CFD), Reynolds-averaged Navier-Stokes today and, probably, large eddy simulation in the future. It is worth mentioning, however, that increased reliability with CFD is not automatically achieved. There are important issues related to the boundary conditions applied, the turbulence modeling and the meshing, which in connection with the flow physics, may strongly influence the quality of the results. Also, modeling of wake effects in complex terrain is quite challenging since the basic approximations of the actuator disk theory have been devised for uniform inflow in flat terrain and break down when a machine is located in the wake of another and/or in complex terrain. Hence, the focus of the work being carried out in this part of UpWind Integrated Project is dual: (i) to evaluate the performance of CFD models in large wind farm applications in complex terrain and (ii) to shed some light in the development of the wakes in a complex terrain environment.

\section{THE METHODS}

Dealing with complex terrain, the application of inherently non-linear CFD models in the resource assessment studies potents enhanced prediction accuracy when compared with the linearized state-of-the-art commercial models ${ }^{5}$ [Wind Atlas Analysis and Application Program (WAsP) ${ }^{6}$ and MS-Micro, ${ }^{7}$ for instance]. However, its use has been limited, mainly because of the increased computational requirements that render those methods impractical for application in a daily basis.

Wind energy development in complex terrain areas has revealed the critical issues that underlie complex terrain flows: Steep mountain ridges contribute to severe flow turning and wind rose narrowing, which are relaxed as the distance from the ground increases. In extreme cases, an inversion of the wind shear and a downstream flow separation is also possible, which are followed by an increase in the turbulence intensity, that renders not all locations on a mesa appropriate for W/T siting. Finally, the flow variation in complex terrain is remarkably high and calls for multimast campaigns, which increases the total costs and lengthens the wind farm design periods.

In this context, CFD solvers have been adapted to simulate the atmospheric boundary layer and to be used for flow prediction in complex terrain. The first step taken was to validate the Navier-Stokes solvers against measurements ${ }^{8,9}$ before applying them in W/T siting. ${ }^{10,11}$ The main pitfall of these models lies in the turbulence modeling, which is calibrated in aerodynamic (wind tunnel) rather than atmospheric conditions to allow its straightforward use in atmospheric flows. It is only very recently that atmospheric flow modeling was attempted using a large eddy simulation technique. ${ }^{12}$ At the same time, wake modeling was restricted in flat terrain applications ${ }^{13}$ with simpler, or more advanced, wind farm models being mainly developed for offshore wind energy applications. ${ }^{14,15}$

By recognizing the importance of accurate wind flow modeling in large wind farms located in complex terrain, two Navier-Stokes solvers are used in this paper for flow simulation and power prediction in complex terrain applications. In both solvers, a W/T is modeled as a momentum absorber through its thrust coefficient by imposing a uniform distribution of axial forces over the rotor disk. The two Navier-Stokes solvers are briefly presented below.

\subsection{CRES-flowNS}

CRES-flowNS ${ }^{16}$ is a full three-dimensional (3D) Navier-Stokes solver with a $k-\omega$ turbulence closure, suitably modified for neutral atmospheric conditions. The momentum equations were numerically integrated introducing a matrix-free pressure correction algorithm that maintains the compatibility of the velocity and pressure field corrections. Discretization was performed with the finite volume technique using a body-fitted coordinate transformation on a curvilinear mesh. Convection terms were handled by a second-order upwind scheme bounded through a limiter, whereas centered second-order schemes were employed for the diffusion terms. Velocity-pressure decoupling was prevented by a linear fourth-order dissipation term added into the continuity equation.

The modified constants for neutral atmospheric conditions of the standard $k-\omega$ turbulence model are as follows: ${ }^{17}$

$$
\alpha=0.3706, \beta=0.0275, \beta_{*}=0.033, \sigma=0.5, \sigma_{*}=0.5
$$

Turbulence modeling in CRES-flowNS was augmented to account for the significant underestimation of the near-wake deficit that has been reported in W/T simulations. ${ }^{18,19}$ Several alternatives have been suggested to delay the wake flow recovery. ${ }^{20}$ However, their main deficiency is their dependence upon constants that need tuning with experimental data. 
To ameliorate this anomaly, a concept already applied in stagnation point aerodynamic flows, ${ }^{21}$ where a similar behavior of the two-equation turbulence models has been observed, was introduced in CRES-flowNS. It is based on the global 'realizability' constraint for the turbulent kinetic energy, $k, 2 k \geq \overline{u^{2}} \geq 0$, where $u$ can be any component of the fluctuating velocity. By applying this constraint on the eddy-viscosity formula written in the principal axes of the strain tensor, the following bound for the turbulent time scale is obtained:

$$
T=\min \left(\frac{1}{\omega}, \frac{2}{3} \sqrt{\frac{3}{8 S^{2}}}\right)
$$

where $S^{2}=S_{i j} \cdot S_{j i}$ and $S_{i j}$ is the strain tensor given by the following relationship:

$$
S_{i j}=\frac{1}{2}\left(\frac{\partial U_{i}}{\partial x_{j}}+\frac{\partial U_{j}}{\partial x_{i}}\right)
$$

with $x_{i}$ being the Cartesian coordinates and $U_{i}$ being the velocity components. Equation (3) can be used to substitute the turbulent time scale in the calculation of the turbulent viscosity and the $\omega$ (or $\varepsilon$ ) transport equation. Because of its general assumptions, this constraint can be applied to any flow simulation, even in atmospheric flows; its main advantage being not requiring calibration using data.

The effect of this near-wake turbulence limiter on the turbulence viscosity is shown in Figure 1, where the predictions of the CRES-flowNS solver for a single W/T simulation in flat terrain are depicted with and without correction. As expected, the effect of the limiter (reduction of the level of the turbulence viscosity) is significant in the near wake and attenuates in the far wake.

\subsection{CFDWake}

CFDWake is an elliptic CFD wake model, ${ }^{22}$ based on the coupling between the actuator disk technique and CFD wind modeling, implemented through the commercial software package FLUENT (Ansys, Inc., Canonsburg, Pennsylvania). Once the grid is generated, the steady-state 3D Navier-Stokes equations are solved: the continuity equation, the momentum conservation equations in the three directions and the transport equations for $k$ and $\varepsilon$. A second-order upwind discretization
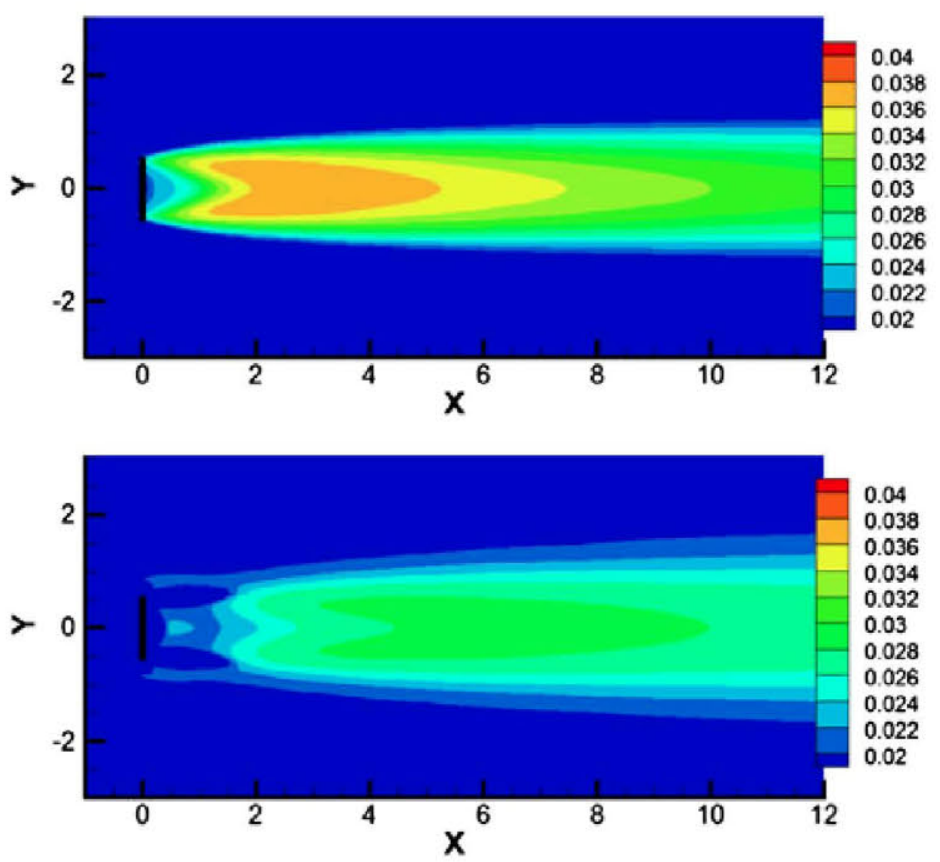

Figure 1. Effect of the near-wake turbulence limiter on the turbulent viscosity for a single W/T simulation. Contours are depicted on the $x-y$ plane at the hub height of the turbine. The W/T rotor disk is located at $x=0$ and denoted with a thick black line. Upper: calculations using the standard $k-\omega$ turbulence model. Lower: calculations applying the correction to the standard $k-\omega$ turbulence model. 
scheme based on multilinear reconstruction approach was used for all dependent variables. The standard $k-\varepsilon$ turbulence model was used with modified constants adapted to the features of the surface boundary layer: ${ }^{23}$

$$
C_{1 \varepsilon}=1.176, C_{2 \varepsilon}=1.92, C_{\mu}=0.033, \sigma=1.0, \sigma_{*}=1.3
$$

\subsection{Boundary conditions}

Both models use the vertical profiles of the fully developed turbulent surface boundary layer as free-stream conditions at the inlet of the computational domain. ${ }^{17}$ The inflow wind speed profile follows the logarithmic law:

$$
U_{x}=\frac{u_{*}}{K} \ln \left(z / z_{0}\right)
$$

where $u_{*}$ stands for the friction velocity, $K$ is the von Karman constant and $z_{0}$ is the roughness length. The inflow profile of $k$ is given by the following relationship: ${ }^{24}$

$$
k=\frac{u_{*}^{2}}{\sqrt{\beta_{*}}}
$$

where according to Equation (1), $\beta_{*}=0.033$.

The respective profiles for $\omega$ and $\varepsilon$ are as follows:

$$
\omega=\frac{u}{\sqrt{\beta_{*}} \cdot K \cdot z}, \varepsilon=\frac{u_{*}^{3}}{K \cdot z}
$$

Neumann conditions (zero gradients) were imposed for all quantities at the top and lateral boundaries. At the outlet plane, CRES-flowNS also applies Neumann conditions, whereas CFDWake imposes an atmospheric pressure value. Both codes use wall functions close to the ground. CRES-flowNS demands that the logarithmic profile of Equation (5) is maintained at the first grid point above ground. CFDWake adapts the standard wall functions by setting a link between the turbulent law of the wall modified for mechanical roughness and the surface boundary layer log-law based on the roughness length.

\section{4. $\mathrm{W} / \mathrm{T}$ modeling}

Both models consider the rotor disks of the W/Ts as momentum absorbers upon which a uniform distribution of axial forces is applied. The axial force that the W/Ts exert over the incoming flow is prescribed from the actuator disk theory, and it is calculated through the thrust coefficient for a corresponding upstream wind speed:

$$
F=0.5 \rho U_{\mathrm{ref}}^{2} C_{T} A
$$

In Equation (8), $\rho$ is the air density, $U_{\text {ref }}$ is the reference wind speed for the thrust coefficient calculation, $C_{T}=C_{T}$ ( $\left.U_{\text {ref }}\right)$ is the thrust coefficient and $A$ is the surface area of the rotor disk. The rotor disk of each W/T is discretized by a number of control volumes. Each control volume acts as a momentum sink through the actuator force estimate by Equation (8).

In flat terrain cases, the rotor disks of the W/Ts are perpendicular to the main flow, and they lie on the $y-z$ planes of the computational grid. However, in complex terrain due to the topography effects, the discretization of the rotor disks must take into account the fact that the orientation of the W/T rotors may not be perpendicular to the main flow direction (the flow direction at the inlet plane of the computational domain). To this end, CRES-flowNS performs a first computation without W/Ts (including only the terrain topography) to estimate the yaw angle at each rotor disk. Assuming operation with no yaw misalignment, the predicted wind direction at the hub height at each W/T position provides an estimation of each W/T's orientation. Next, the discretization of the rotor disks is carried out using the grid cells that fulfill certain geometrical criteria regarding the W/T's orientation and the distance from the rotor center.

Apart from the correct alignment of the rotors, an additional challenge in the modeling of W/T wakes in real wind farm simulations in complex terrain is the accurate application of Equation (8). Stemming from the definition of the thrust coefficient from single W/T operation in flat terrain and uniform flow conditions, Equation (8) requires the reference wind speed as input, which in flat terrain applications can be easily defined at the location of the W/T, at hub height, through a measurement campaign. Since there are no topographic effects in flat terrain, the same wind speed value can be used as reference in the relevant simulations because the area affected by the presence of a W/T is restricted to one or two rotor diameters $(D)$ upwind of the rotor. It should be pointed here that apart from the modeling of the wakes, the choice of the reference wind speed, directly affects the power production estimation from an individual W/T in a wind farm and, consequently, the wind farm in total. 
Unfortunately, such an approach cannot be followed in cases that the flow field upstream of a W/T is affected by the terrain and/or by the wakes of upstream or neighboring W/Ts. In this respect, from a methodological point of view, a run of the model without the W/Ts is first required to provide the accurate wind conditions at a W/T position followed by a second run in which the momentum absorbers representing the W/T rotors are activated. This approach is utilized in CFDWake, and in the case of a wind farm with $N$ rows, it requires $(N+1)$ sequential runs to be carried out:

- First, a run is performed without W/Ts to estimate the free-stream wind speed values at the W/T positions in the first row.

- These values are used to prescribe the corresponding sink terms defined by Equation (8), using as reference wind speed value the one obtained from the first simulation at the center of the rotor disks of the W/Ts in the first row; in a second run, that includes only the first row of W/Ts.

- The resulting wind speed values at the positions of the W/Ts in the second row are then used as the reference wind speeds, and the corresponding sink terms are estimated and activated in a third run, which includes the first and second rows of W/Ts.

- This process is made until the last row is reached.

In this way, the simulation operates in a hybrid parabolic-elliptic mode, which renders it impractical, at least for application on a daily basis, since this approach can be effectively applied only in cases that the flow is nearly perpendicular to the rows of W/Ts; on the contrary, in any other case, it would require a number of steps equal to the number of the W/Ts in the simulated wind farm.

To ameliorate this methodological pitfall, two alternatives were tested in the CRES-flowNS method, which require one single computation for any wind farm. The first one employs, as reference wind speed, the value at $1 \mathrm{D}$ upwind of each rotor at hub height, without introducing any correction to account for the ellipticity of the flow field and the consequent reduced reference wind speed.

The second is based on the definition of the induction factor:

$$
a=\frac{U_{\text {ref }}-U_{\text {disk }}}{U_{\text {ref }}}
$$

where $U_{\text {ref }}$ is the, unknown, W/T reference wind speed and $U_{\text {disk }}$ is the wind speed at hub height at the location of the $\mathrm{W} / \mathrm{T}$, or the average wind speed over the disk surface. The W/T thrust coefficient is expressed as a function of the induction factor: ${ }^{25}$

$$
C_{T}= \begin{cases}4 a(1-a), & a<0.4 \\ 0.89-\frac{0.20-(a-0.143)^{2}}{0.643}, & a \geq 0.4\end{cases}
$$

and Equations (9) and (10) along with the thrust coefficient curve $C_{T}=C_{T}\left(U_{\text {ref }}\right)$ can be solved iteratively to provide the $U_{\text {ref }}$ value. This method has the advantage that the estimation of $U_{\text {ref }}$ is not linked to the determination of a certain distance upstream of the W/T. However, it bears potential and uniform flow approximations that are inherent to the induction factor relationships employed. However, from multiwake simulations in flat terrain, ${ }^{20}$ it features a good prediction potential, at least in flat terrain. All three approaches are evaluated in the next section using data from an operating wind farm.

\section{SIMULATION OF A 43 W/T WIND FARM IN COMPLEX TERRAIN}

\subsection{Description of the test case and numerical simulation}

Both models were applied to a large wind farm located in complex terrain in Spain, with 43 machines positioned in five, nearly parallel, rows (see Figure 2). The distance between the first three rows is about $11 \mathrm{D}$, whereas the fourth and fifth rows are further apart, at distances 15 and $22 \mathrm{D}$ from the third row, respectively. The distance between two machines in the same row is $1.5 \mathrm{D}$. It is important to state that 10 out of the 43 machines feature a higher hub height than the others. The thrust and power curves of the W/Ts have been provided from the owner in the context of the UpWind Integrated Project.

The terrain contours and a layout of the surface grid for the examined case, corresponding to a wind direction value of $327^{\circ}$, are presented in Figures 2 and 3, respectively. Figure 3 shows the grid layout in the main flow direction that is nearly perpendicular to the $\mathrm{W} / \mathrm{T}$ rows.

The discretized terrain consists of $351 \times 435$ points. Taking into account that 45 grid lines have been used in the vertical direction, the computational grid consists of nearly 7 million grid points. As depicted by Figure 3 , the inflow boundary of the computational domain has been placed far enough from the locations of the W/Ts, so that the largest possible part of terrain influencing the development of the flow is taken into account. Both models use a computational domain that covers the same area and features similar grid spacing in the three directions. Specifically, the inlet boundary has been positioned 


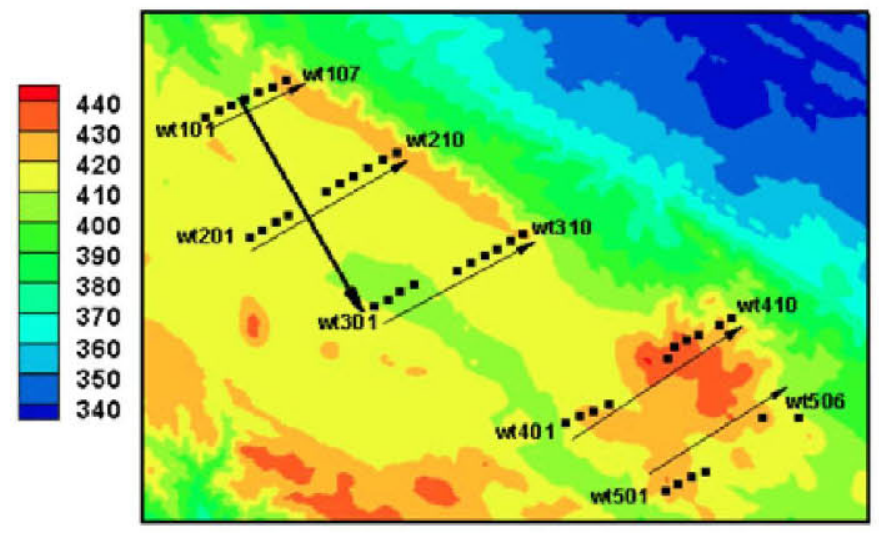

Figure 2. Layout of the complex terrain wind farm. The arrow perpendicular to the W/T rows shows the wind direction of $327^{\circ}$ that is studied in this paper. The colored contours indicate terrain elevation (meters above sea level).

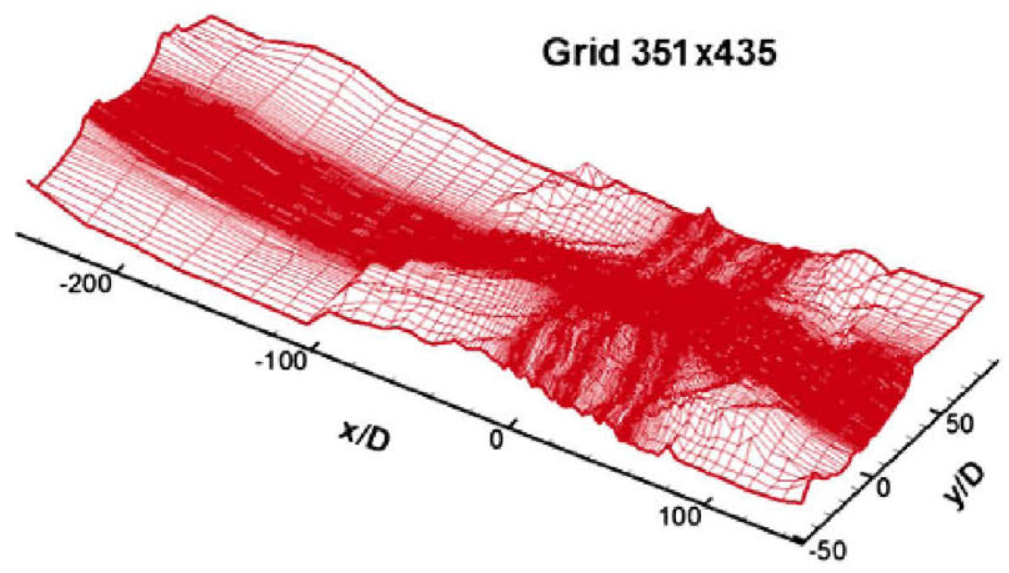

Figure 3. Layout of the surface grid for the $327^{\circ}$ case studied.

approximately $11 \mathrm{~km}$ upstream of the first W/T row, whereas the outlet boundary has been positioned approximately $4 \mathrm{~km}$ downstream of the last W/T row. Each side boundary is located at a distance of $2 \mathrm{~km}$ from the closest W/T. The grid spacing in the $x-y$ plane starts from a minimum value of $0.1 \mathrm{D}$ at the locations of W/Ts and increases outwards using a geometrical progression with ratio 1.15 . In the vertical direction, the first grid line has been positioned at $0.5 \mathrm{~m}$ above ground.

To achieve grid independent results, a second computational mesh is generated and incorporated in CRES-flowNS, which is finer in the area of the wind farm. In general, high computing requirements for CFD models make it impossible sometimes to evaluate the models in such large wind farms. To this end, the second, refined, grid covers only the first three rows of W/Ts in the wind farm. In this grid, the minimum value of grid spacing, close to the W/T rotors, has been reduced to $0.07 \mathrm{D}$ in $x$-direction and to $0.05 \mathrm{D}$ in $y$-direction. In the vertical direction, the first grid line has been positioned at $0.25 \mathrm{~m}$ above ground.

In addition to the simulations with CFD, power production results for the specific wind farm were generated using WAsP, which is the industry standard approach. WAsP is based on a linearized model used in the European Wind Atlas. ${ }^{27}$ The WAsP program ${ }^{6}$ uses meteorological data from a measurement station to generate a local wind climate from which the effects of obstacles, roughness and complex terrain have been removed. To produce a wind climate for a nearby wind farm or W/T site, wind speed and direction are fitted to a Weibull distribution, and these local effects are reintroduced for the new site. In terms of wind farm modeling, ${ }^{2}$ the wake model assumes a 'top-hat' wake shape and a linear expansion of the wake where the rate of expansion is set by the wake decay coefficient $k$. The velocity deficit $\Delta U$ is calculated using the following:

$$
\Delta U=U_{0}\left(1-\sqrt{1-C_{\mathrm{T}}}\right)\left(\frac{D}{D+2 k X}\right)^{2} \frac{A_{\text {overlap }}}{A}
$$


where $U_{0}$ is the free-stream wind, $C_{\mathrm{T}}$ is the thrust coefficient, $D$ is the rotor diameter, $X$ is the downwind distance between the turbines, $A$ is the area of the rotor plane of the downwind turbine and $A_{\text {overlap }}$ is the overlapping area of the upwind wake and $A$.

The main advantage of the program is that it is fast and robust. It does not predict flow separation in complex terrain, even if terrain is sufficiently steep, although there are methods for improving its predictions in very complex terrain. ${ }^{28}$ WAsP parameterizations are not designed for the near-wake region (less than $3-4 \mathrm{D}$ ) downstream. WAsP is being run here with a standard wake decay coefficient of 0.075 regardless of the turbulence intensity. The wake decay coefficient defines wake expansion, which is related to turbulence intensity, so using one standard value for all simulations has an impact on the final results. Finally, WAsP has a linear treatment of complex terrain and wake losses where the flow is calculated for the terrain and wake losses are added to these results.

For the WAsP simulations in the complex terrain wind farm, the same topographic grid was used as for the CFD models, but it was subject to contouring and did not have the fine resolution close to the first three rows. The simulations were made for narrow direction bins $\left(327 \pm 5^{\circ}\right)$ with wind speeds distributed between the $7-8 \mathrm{~m} \mathrm{~s}^{-1}(10 \%)$ and $8-9 \mathrm{~m} \mathrm{~s}^{-1}(90 \%)$ bins, giving a mean wind speed close to $8.4 \mathrm{~m} \mathrm{~s}^{-1}$. This results in an unrealistic Weibull shape parameter, and thus, the program is being used in a way that is not recommended.

\subsection{Uncertainties of the comparison between predictions and measurements}

The operational power data considered herein have been selected and averaged to correspond to a 'free-stream' wind speed of $8 \pm 0.5 \mathrm{~m} \mathrm{~s}^{-1}$ for the first W/T in the first row, which is the reference turbine for the definition of the test case and is denoted as wt101 in Figure 3. The actual power productions are averaged from data covering the range $327^{\circ} \pm 5^{\circ}$ of wind directions for the reference $\mathrm{W} / \mathrm{T}$. There are a number of specific issues relating to the comparison of wake predictions and measurements:

- Establishing the free-stream flow. In the present case, it is assumed that the wind direction at the inlet plane of the computational domain is the same with the one in the reference W/T, without considering the topographic effects between the inlet plane and the location of wt101. Also, for determining the wind speed, the power measurements are used without adjusting the power curve of the W/Ts for the specific site meteorological conditions.

- Wind direction, nacelle direction and yaw misalignment make it difficult to establish accurate and coherent data, considering the fact that wind vanes may have a bias of up to $5^{\circ}$. In such a large wind farm, each turbine may have a separate bias on the direction, which is very difficult to determine from the operational data.

- Natural fluctuations in the wind speed and direction. Whereas wake measurements incorporate a degree of natural variability, model simulations are made for specific wind speed and directions. In the same line, the operational data do not provide any information about the atmospheric stability but represent both summer and winter, day and night periods. On the contrary, simulations are made for neutral stability conditions.

- Wake transport time through the wind farm. In such a large wind farm context, the time scale of wake transport must be considered. The specific wind farm has a length of nearly $4 \mathrm{~km}$. At a wind speed of $8 \mathrm{~m} \mathrm{~s}^{-1}$, the travel time through the array is more than $8 \mathrm{~min}$. The wind direction will be subject to natural fluctuations in addition to possible wake deflection, but there will also be natural variations in the wind speed over this time scale. On the contrary, steady-state CFD simulations assume perfect resource coherence in all the area modeled.

\subsection{Results}

A first comparison of the flow field with and without W/Ts is illustrated in Figure 4, where predictions of the wind speed at hub height in the direction of the flow are presented for the seven machines of the first row, starting from $20 \mathrm{D}$ upstream to $10 \mathrm{D}$ downstream. Results from both CRES-flowNS and CFDWake are included in the figure. The comparison concerns only the seven turbines in the first row, which are experiencing free-stream conditions. The first conclusion drawn, related to the validation of the two methods, is that both of them produce similar results in the case without turbines and in the region free of the turbines in the computations with W/Ts. The small differences arising can be attributed to the differences in the discretization of the topography and the turbulence closure used. In addition, both methods predict that the upstream influence of each machine to the flow starts from at least $6 \mathrm{D}$ upstream of the turbine rotor, which is certainly bigger than in flat terrain.

The wind speed deficit predicted by CRES-flowNS is higher compared with the one by CFDWake. This is anticipated since in CRES-flowNS computation, the effect of the near-wake turbulence correction is to increase the velocity deficit close to the W/T region. In addition, differences arise from the fact that CRES-flowNS utilizes, as reference wind speed, the velocity $1 \mathrm{D}$ upstream of the machines at hub height. This is considered to be the optimum fixed distance for the reference velocity definition, when a W/T is located in the wake of another, and has been widely used in flat terrain calculations. 

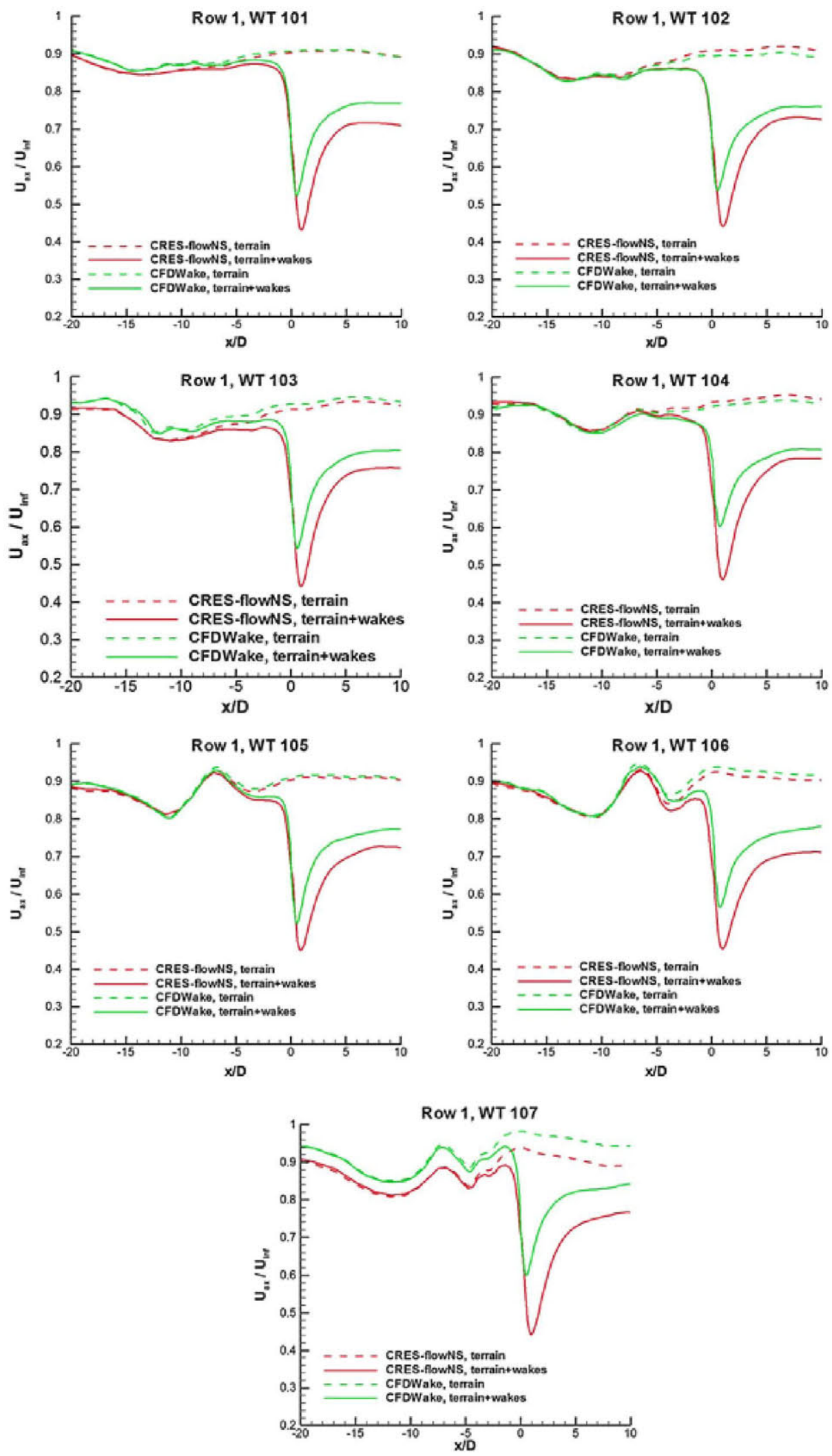

Figure 4. Simulated wind speed at hub height with and without W/Ts for the seven W/Ts in the first row. 
However, as depicted in Figure 4, this wind speed is lower than the wind speed at the location of the turbines (when the W/Ts are absent).

A second remark concerns the effect of the topography on the flow angle, which determines the yaw of the W/Ts. The flow angles at the W/T locations are presented in Figure 5, as predicted from the simulation without the turbines using CRES-flowNS. It is noted that in the wind farm computations with CRES-flowNS, this misalignment of the W/Ts is taken into account to position the rotor disk. For the first three rows, two distributions are presented, one using the coarse grid and a second one using the finer grid. Comparison of the two distributions shows a good agreement between the results using the two grids. For these rows, maximum flow angles do not exceed $-5^{\circ}$ at all the W/T locations. However, at the back rows, flow angles increase significantly, reaching up to $-15^{\circ}$ as a result of the topography. Again, it is noted that these simulations are steady-state, so they cannot account for unsteady effects as the meandering of the wake, and so on.

In Figure 6, the predicted power productions from the W/Ts in the first four rows are compared with the operational data. Results for only the first four rows are provided, since these are the turbine rows that have been simulated using CFDWake. The normalization of power for each and every $\mathrm{W} / \mathrm{T}$ is carried out using the average power value from the seven machines in the first row. The operational data are accompanied by their uncertainty, which is estimated as one standard deviation. Four sets of CFD predictions, three using the CRES-flowNS and one using the CFDWake model, are depicted in the figure. The distributions denoted with 'CRES-flowNS, terrain' refer to the power predictions without W/Ts that include only the effect of the topography (including the effect of the different hub height in some W/Ts), as predicted using the CRESflowNS model. The power production calculations in the presence of W/Ts using the three different approaches described
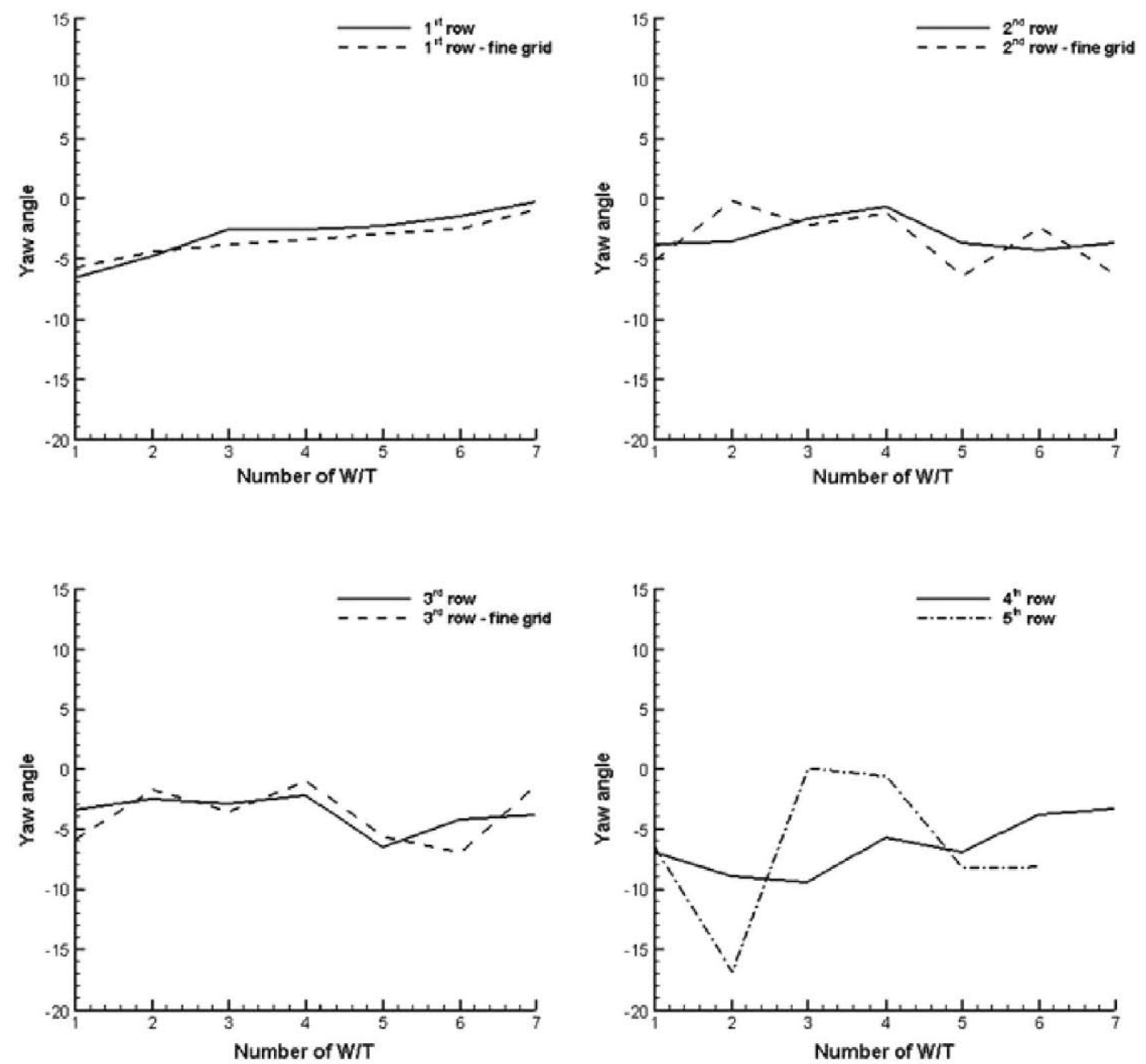

Figure 5. Flow deviation from the main direction $\left(327^{\circ}\right)$ at the W/T locations. For the first three rows, the two lines depict results from the simulation with the grids described in Section 3.1 where the fine grid includes a refinement in the area of the first three W/T rows $(0.07 \mathrm{D}$ in the $x$-direction, $0.05 \mathrm{D}$ in the $y$-direction). Calculations have been performed using the CRES-flowNS solver. 

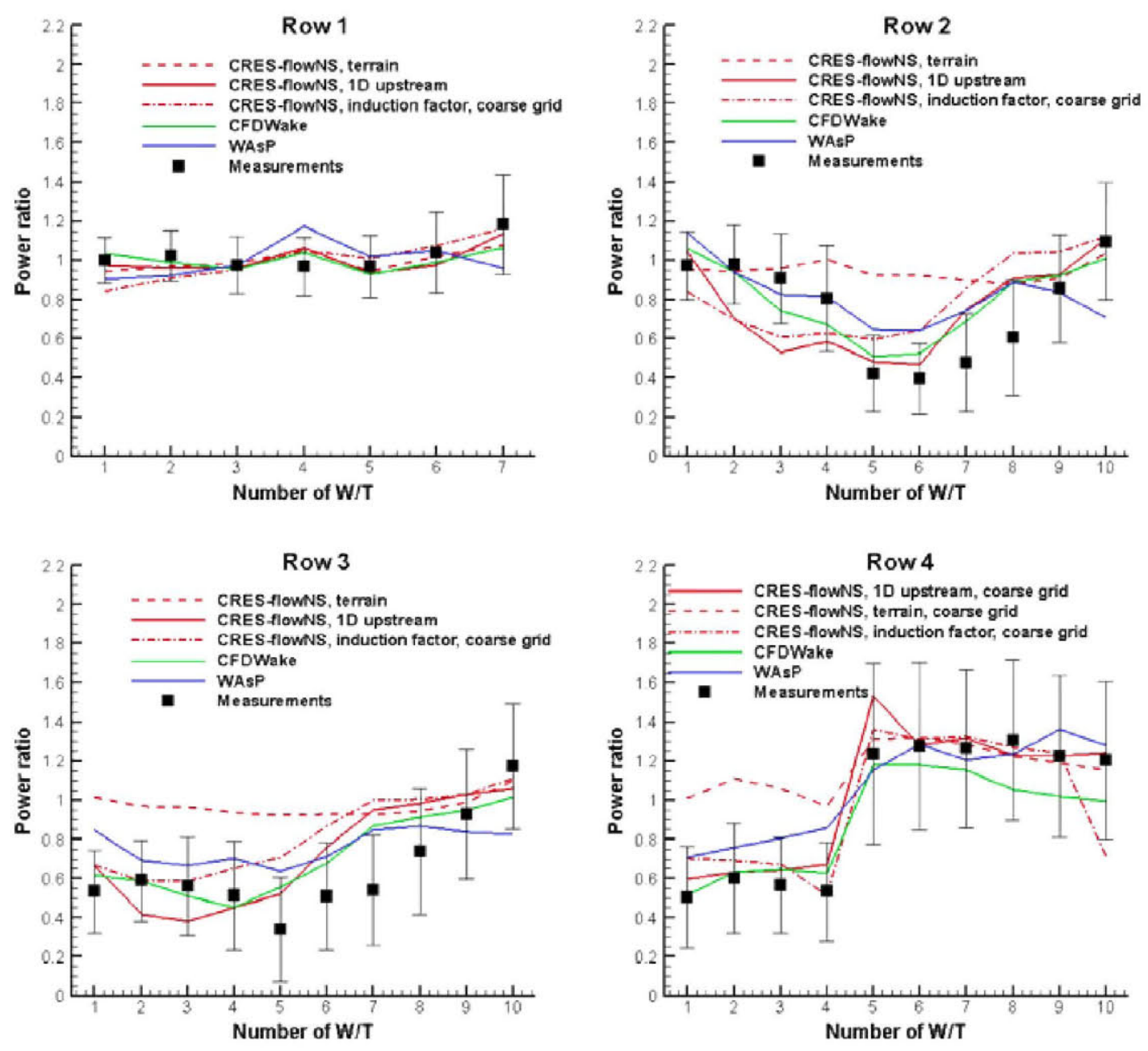

Figure 6. Power ratios of the $W / T s$ in the four rows, with reference to the average power of the seven $W / T s$ of the first row, for the complex terrain wind farm for wind direction $327^{\circ}$. CRES-flowNS, CFDWake and WAsP predictions are compared with operational data.

in the previous paragraph are presented in the graphs: The baseline method is denoted in the figure as 'CFDWake', whereas the results using the $1 \mathrm{D}$ upstream value of the wind speed and the induction factor method are denoted as 'CRES-flowNS, 1D upstream' and 'CRES-flowNS, induction factor', respectively. It must be noted that simulation using the induction factor method has been carried out only using the coarse grid, whereas for the fourth row, results of CRES-flowNS model refer to the coarse grid since the fine grid simulation covered only the first three rows. In addition to the CFD results, the predictions of WAsP are included in the same figure for all machines in the first four rows.

A first observation is that the uncertainty of the operational data is high in all rows, including the first one; this highlights the difficulty in extracting coherent datasets from such large wind farms. Predictions by WAsP tend to have less variability than the observed power or the predictions from the CFD model but broadly reproduce the power variations along the turbine rows. It must be noted that in such complex terrain, utilization of WAsP may be close to its limits; this is the reason why it cannot well reproduce the difference caused by the terrain effect between the first and second groups of turbines in the fourth row, which is dominant in that region. Also, the WAsP flow simulations are run for a sector of $10^{\circ}$ centered on $330^{\circ}$.

Because of the proper estimation of the reference wind speed, the predictions using CFDWake exhibit a better agreement with measurements, with the exception of the second group of W/Ts in the fourth row. The predictions of CRES-flowNS using the $1 \mathrm{D}$ upstream value of the wind speed are qualitatively in fair agreement with the predictions of the CFDWake and the operational data. It must be pointed here that power predictions by each model have been non-dimensionalized using the average power from the seven machines in the first row from the same simulation; so, all predictions feature a 
unity average value for the first row. A direct consequence is that the underestimation of the reference wind speed in the simulation using the $1 \mathrm{D}$ upstream value is not reflected in the power production graphs.

The use of CFD improves predictions as confirmed by the comparison of the mean root mean square (RMS) errors for the fourth row (see Table I). For the other three rows, according to the same table, the CFDWake predictions present the smallest mean error, whereas both CFDWake and CRES-flowNS using the 1D upstream approach are still in a closer agreement with the measurements. However, their differences with WAsP have been reduced because the effect of the complex terrain is not dominant. The large errors of the CRES-flowNS using the induction factor approach are partly attributed to the coarser grid but also indicate that the applicability of this method in complex terrain demands further investigation.

As expected, the power production predictions for the turbines in the first row are in good agreement with the power predictions without the turbines because these machines operate in free-stream conditions without any wake effects. An exception to this is the power ratios obtained using the induction factor method, but for this row, the differences between the operational data and the predicted power ratios are small. The power reduction for most of the W/Ts at the second and third rows in comparison with the power estimations without the turbines comes from the interaction with the upstream W/Ts as a result of the wake effects. At the fourth row, the second group of six W/Ts is not significantly affected by the wakes of the preceding turbines, because they are located almost outside of the area that the wakes from the preceding rows develop. In addition, they are located at higher terrain altitudes where the topography effect is dominant. By comparing the results by the three different methods tested, it is obvious that the induction factor method results in an overestimation of the power production for all machines, with the exception of the fourth and tenth machines in the fourth row, where the predictions are considerably lower, indicating again that the use of this method in complex terrain should be further investigated.

The comparison of the predictions with the operational data, in the first row, is fair for all but the fourth machine, where the increase in the hub height is not reflected in the operational data. However, significant deviations are observed for the second group of W/Ts at the second and third rows. Apart from possible operation of these machines with yaw misalignment and the large uncertainty of the experimental data, computational reasons that drive the differences could be (i) insufficient terrain discretization and (ii) an erroneous estimation of the reference wind speed for the thrust and power calculation. Regarding (i), it should be stressed that grid independence was achieved at least for the grid density over the rotor disk surfaces (100 grid points proved to be sufficient for grid independent predictions). However, it was not completely achieved for the grid density over the terrain even with the finer grid generated. Regarding (ii), it seems that the coupling of the CFD solver to a blade element momentum (BEM) solver is required to correctly model the W/T within a CFD solver. However, apart from the increase in computational requirements, the biggest difficulty in such approach lies in the need for detailed W/T description in terms of its rotational speed, the geometry of the blades and the aerodynamic polars of the airfoils in the span of the blades.

\section{WAKE DEVELOPMENT IN COMPLEX TERRAIN}

In the previous section, the issues that underlie the accurate modeling of the flow in large wind farms in complex terrain were discussed. It was shown that CFD simulations of this type require big meshes of several million grid points resulting in a significant increase of the computational cost. Computing demands are considered no longer excessive, because of the fast computer development that occurred during the last decades. However, the best compromise between cost and accuracy is always sought, indicating that straightforward engineering-type models are still desirable, if they can be properly calibrated to produce acceptable results. So, from a practical point of view, (large) wind farms in complex terrain are still designed by employing results for the wind resources (obtained using the linearized state-of-the-art commercial model $\mathrm{s}^{5}$ as WAsP $\mathrm{P}^{6}$ and MS-Micro, ${ }^{7}$ or even CFD solvers ${ }^{10}$ ) that do not include the interaction of the W/Ts. Wake effects are added through semi-empirical, engineering-type models. ${ }^{26}$

These models have been calibrated to predict the wake characteristics in flat terrain, and the question arising is if they can be calibrated or even modified to predict the wake characteristics in complex terrain. To answer this question, a first step is the comparison of wake characteristics between flat and complex terrain. Such a comparison may shed some light

Table I. Comparison of the mean RMS errors of the models for each W/T row. The two CRES-flowNS models represent (a) the 1D upstream method and (b) the induction factor method.

\begin{tabular}{lcccc}
\hline Model & Row 1 & Row 2 & Row 3 & Row 4 \\
\hline CFDWake & 0.04907 & 0.23578 & 0.29482 & 0.11990 \\
CRES-flowNS (a) & 0.06207 & 0.30044 & 0.36956 & 0.12051 \\
CRES-flowNS (b) & 0.09036 & 0.42751 & 0.49272 & 0.19217 \\
WAsP & 0.09545 & 0.32927 & 0.40523 & 0.26584 \\
\hline
\end{tabular}


to the effect of the topography on the wake development and the possibility of isolating this effect and linearly superimposing it to the deficit and/or the other wake characteristics over flat terrain. In addition, it can provide useful information about the effect of various parameters such as the ambient turbulence, the wind direction and the terrain shape on the wake development over complex terrain.

Within Upwind Integrated Project, the comparison of the wake characteristics between flat and complex terrain was investigated using idealized simulations of a single wake in the case of Gaussian hills. The conclusions deduced from the analysis of the axisymmetric and quasi-3D Gaussian hills can be extended to more complex terrain where the irregularities of the topography can be seen as separate hills.

\subsection{Numerical simulation of the Gaussian hill case}

A Gaussian quasi-3D hill geometry is defined by the following relationship:

$$
z=h \exp \left[-0.5\left(\frac{x}{\sigma}\right)^{2}\right], \sigma=L / 1.1774
$$

where $x$ and $z$ are the horizontal and vertical coordinates, respectively, $h$ is the height of the hill and $L$ is defined as $x(z=h / 2)$. In the case of the axisymmetric hill, the quantity $\sqrt{x^{2}+y^{2}}$ replaces $x$ in Equation (12). The axisymmetric
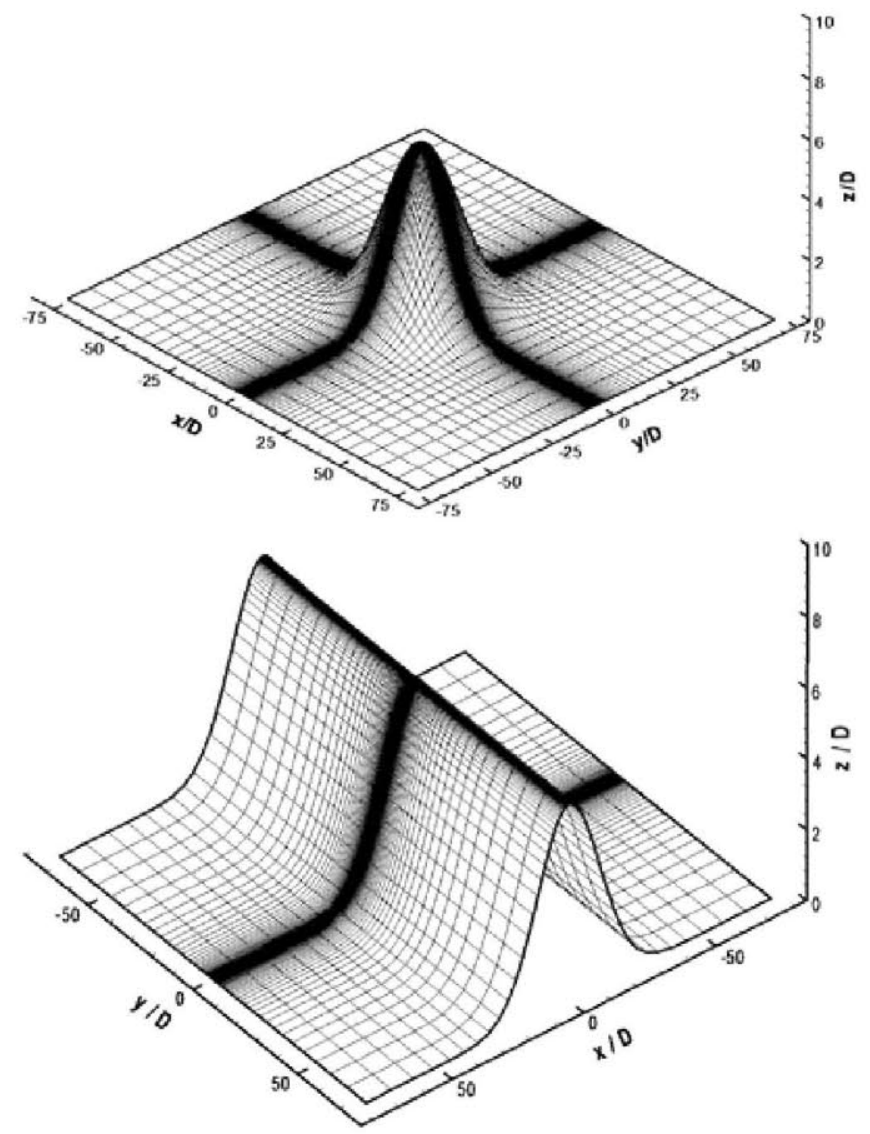

Figure 7. Digitized terrain of the axisymetric (upper) and quasi-3D (lower) Gaussian hill.

Table II. Dependence of the free-stream wind speed on $T I_{\text {in }}$.

\begin{tabular}{lcc}
\hline$T I_{\text {in }}(\%)$ & $z_{0}(\mathrm{~m})$ & $U_{\infty}\left(\mathrm{m} \mathrm{s}^{-1}\right)$ \\
\hline 5 & $2.29 \cdot 10^{-7}$ & 10.90 \\
13 & 0.0445 & 12.47 \\
20 & 0.639 & 13.80 \\
\hline
\end{tabular}


and quasi-3D hill terrains derived from Equation (12) for $L=1750 \mathrm{~m}$ are shown in Figure 7. The configuration investigated corresponds to $h=700 \mathrm{~m}$ and $L=1750 \mathrm{~m}$, which denotes a mean slope of 0.4 . The grid size was about 750,000 nodes for the two hill cases with 101 nodes placed in the streamwise and the lateral directions and 73 nodes in the vertical direction. The disk rotor was discretized using 21 nodes. The corresponding flat terrain cases were discretized using 309 , 000 nodes.

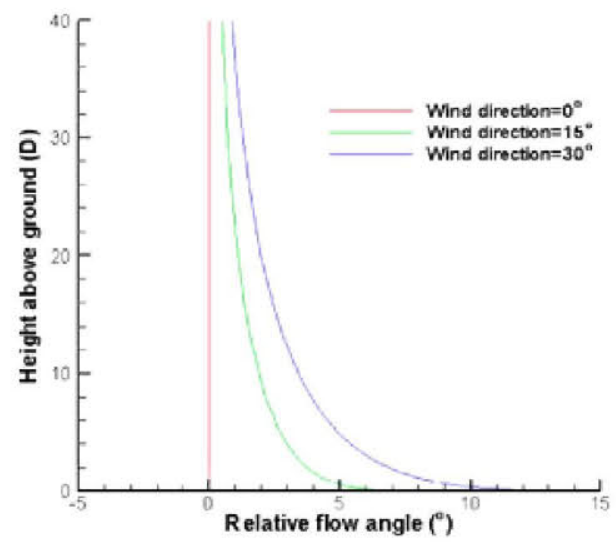

Figure 8. Relative flow angle at hill top in the quasi-3D case.

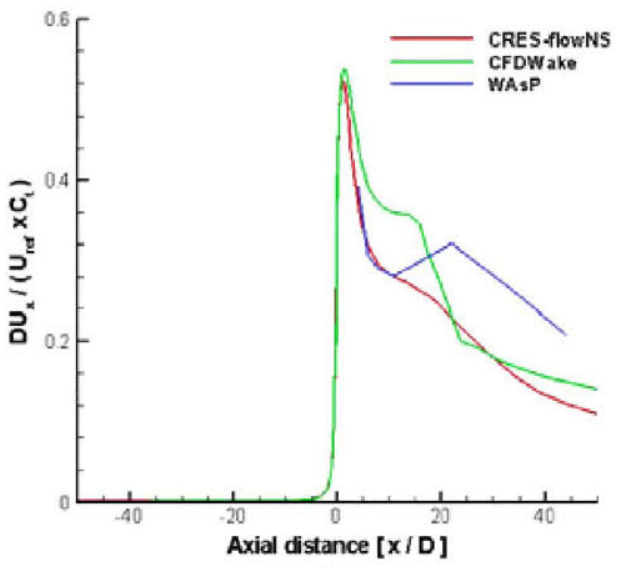

(a)

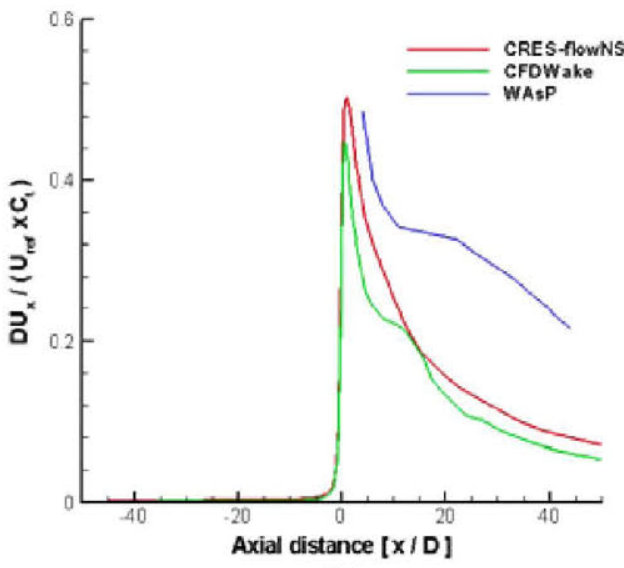

(b)

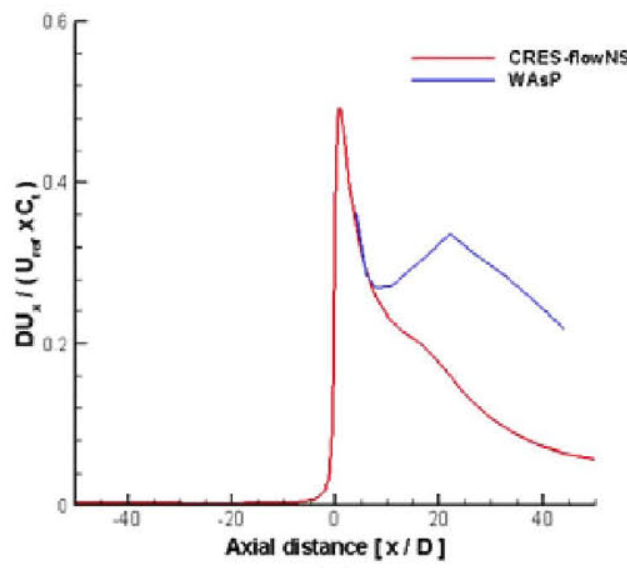

(c)

Figure 9. Deficit variation for the axisymmetric 3D hill. (a) $T l_{\mathrm{in}}=5 \%$, (b) $T t_{\mathrm{in}}=13 \%$ and (c) $T l_{\mathrm{in}}=20 \%$. 
The different configurations were simulated with one $\mathrm{W} / \mathrm{T}$ placed at the hilltop and without. The simulations without the $\mathrm{W} / \mathrm{T}$ are necessary to provide the value of velocity at the W/T position for the calculation of the actuator disk force, as well as the reference velocity for the evaluation of the deficit in the following figures. Actuator disk force is calculated through Equation (8), using the thrust coefficient curve $C_{\mathrm{T}}=C_{\mathrm{T}}\left(U_{\mathrm{ref}}\right)$, which is provided through a BEM method for a range of wind speeds.

The W/T modeled is a paper case $5 \mathrm{MW}$ machine with a diameter of $126 \mathrm{~m}$ and a hub height of $90 \mathrm{~m}$, specifically designed for the purposes of the UpWind Integrated Project. The inflow velocity profile is assumed logarithmic with $500 \mathrm{~m}$ boundary layer height and $10 \mathrm{~m} \mathrm{~s}^{-1}$ velocity at hub height. Three different levels of inlet turbulence intensity $T I_{\text {in }}$ at hub height, 5, 13 and $20 \%$, are examined. The different levels of $T I_{i n}$ correspond to different values of roughness length, according to Prospathopoulos et al. ${ }^{17}$

$$
z_{0}=z_{h u b} \exp \left(-0.9895 / T I_{\text {in }}\right)
$$

and subsequently to different inflow velocity profiles, as depicted in Table II.

For the quasi-3D hill case, the effect of the wind direction was also investigated through simulations for three different wind directions, 0,15 and $30^{\circ}$, where $0^{\circ}$ is perpendicular to hill orientation.

The velocity deficit is calculated with reference to the predictions without $\mathrm{W} / \mathrm{T}$, using the following relationship:

$$
\frac{\Delta U_{x}}{U_{\text {ref }} \cdot C_{\mathrm{T}}}=\frac{U_{a x}(\text { without } W / T)-U_{a x}(\text { with } W / T)}{U_{a x}(\text { without } W / T) \cdot C_{\mathrm{T}}}
$$

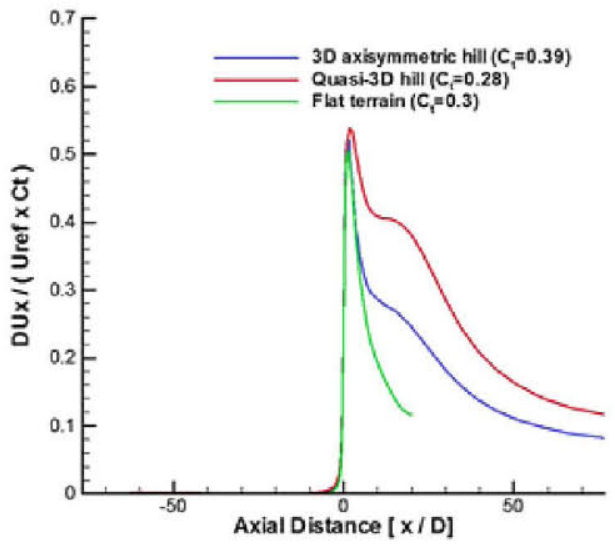

(a)

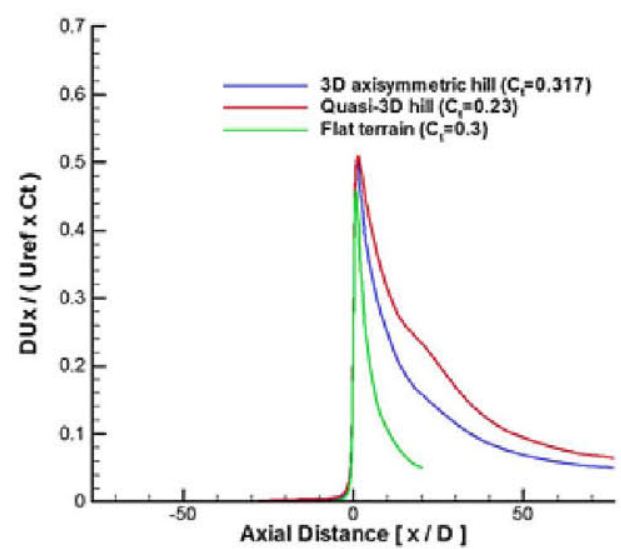

(b)

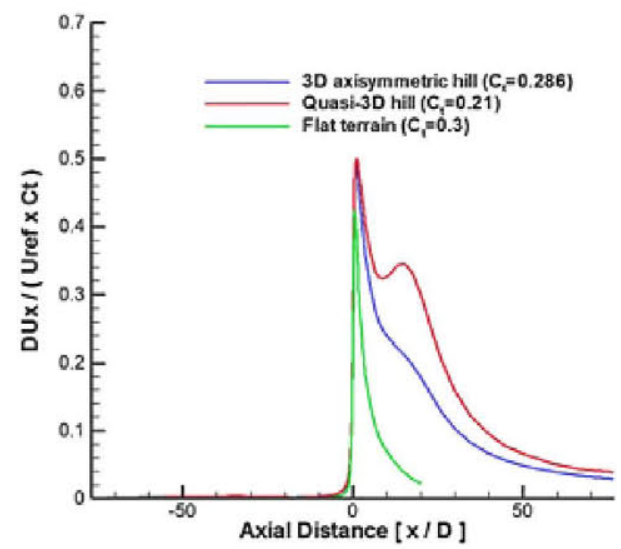

(c)

Figure 10. Comparison of the velocity deficit at hub height among the axisymmetric and the quasi-3D hill and flat terrain. (a) $T I_{1 n}=5 \%$, (b) $T I_{\text {in }}=13 \%$ and $(c) T I_{10}=20 \%$. 
In Equation (14), $U_{a x}$ stands for the $U_{x}$ velocity for $0^{\circ}$ wind direction or the total horizontal velocity $\sqrt{U_{x}^{2}+U_{y}^{2}}$ when the wind direction is 15 or $30^{\circ}$. To assess the effect of the terrain on the velocity deficit predictions, the steps taken are the following:

- The flow field is computed without W/T to provide the reference velocity $U_{\text {ref }}$, at the location of the turbine, at hub height. The thrust coefficient is estimated through the curve $C_{\mathrm{t}}\left(U_{\text {ref }}\right)$ provided by a BEM calculation.

- The flow field is computed by simulating the W/T as an actuator disk force using $U_{\text {ref }}$ and $C_{\mathrm{t}}$ from the previous step.

- The predicted wake characteristics over the hills and the flat terrain are compared.

- The effect of complex terrain is assessed.

Simulations have been carried out using the two CFD solvers and WAsP for the axisymmetric 3D Gaussian hill, where as a further investigation has been performed using CRES-flowNS for both the axisymmetric and the quasi-3D hills. As regards the WAsP simulations for the Gaussian Hill, the direction sector was wider $\left( \pm 15^{\circ}\right)$. The terrain file was built to match the height and length scales used in the CFD simulations. The wake decay coefficient was set to 0.075 , but the roughness of the surface was varied for the three turbulence intensity cases. Wind speeds were divided equally across two wind speed bins 9-10 and 10-11 $\mathrm{m} \mathrm{s}^{-1}$ giving an average wind speed close to $9.9 \mathrm{~m} \mathrm{~s}^{-1}$. This results in an unrealistic Weibull shape parameter, and thus, the program is being used in a way which is not recommended.

\subsection{Results}

The vertical profiles of the relative to the main flow direction angle are presented in Figure 8 at the hilltop for the quasi-3D hill, as they result from the flow field computation without a W/T by CRES-flowNS. It is reminded here that the value of this angle at the hub height of the W/T is used to position the rotor disk in the CRES-flowNS simulation with a W/T.
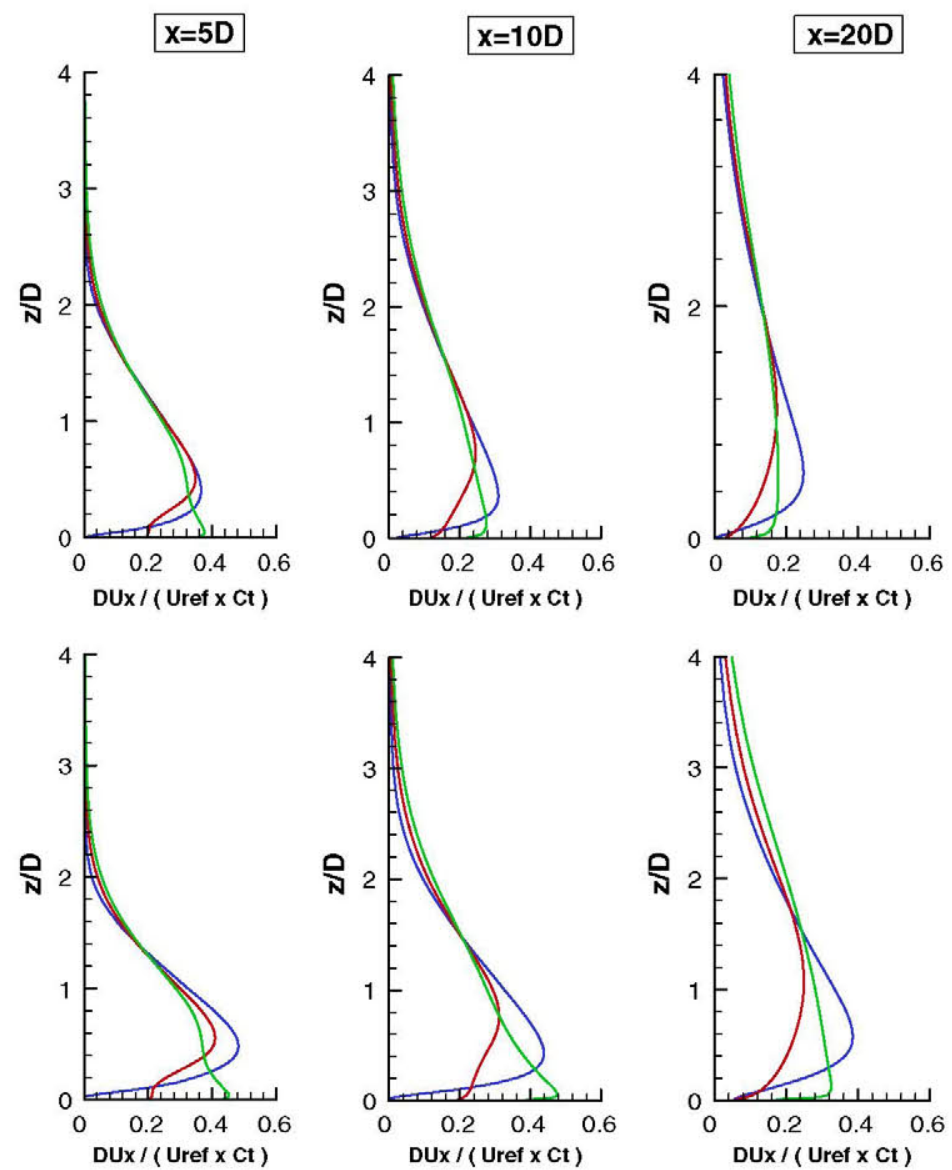

Figure 11. Vertical profiles of velocity deficit at 5, 10 and $20 \mathrm{D}$ downstream the $\mathrm{W} / \mathrm{T}$ for varying inlet turbulence intensities. Blue line, $5 \%$; green line, $13 \%$; red line, $20 \%$. Upper: axisymmetric hill. Lower: quasi-3D hill. 
Profiles of the flow angle are presented for wind direction values of 0,15 and $30^{\circ}$. Obviously in the $0^{\circ}$ case, there is no turning of the flow with the increase of the height above the ground. On the contrary, in the cases where the main flow is not perpendicular to the quasi-3D hill axis, a significant turning of the flow is observed near the ground, more than $10^{\circ}$ in the $30^{\circ}$ case, which is responsible for the wind rose narrowing experienced in complex terrain. As this situation is relaxed with the increase of the height above ground, proper siting of turbines in complex terrain calls for tools that are able to reproduce such pattern when the measuring campaigns do not cover the hub height of the W/Ts.

The predictions of CRES-flowNS, CFDWake and WAsP for the deficit at hub height of the axisymmetric 3D hill are shown in Figure 9. A first observation drawn when comparing the predictions by the three models is that their agreement is better in this case compared with that in the complex terrain wind farm case. WAsP predicts a slower velocity deficit decay in the far wake, especially for $T I_{\text {in }}=13$ and $20 \%$, than the two Navier-Stokes codes that may also be related to the use of a wider direction sector. For $T I_{\text {in }}=5$ and $20 \%$, a close agreement is observed between CRES-flowNS and WAsP predictions up to the distance of $10 \mathrm{D}$. However, after that distance, WAsP predicts an increase in the deficit, which leads to divergence of the predictions at longer distances. The two CFD tools produce very similar results for $T I_{\text {in }}=13 \%$, whereas differences are more pronounced for $T I_{\text {in }}=5 \%$.

By using CRES-flowNS, the velocity deficit is presented in Figure 10 for the 3D axisymmetric and the quasi-3D hills for different levels of $T I_{\text {in }}$. The comparison with the flat terrain case is also shown in the same figure. One important conclusion drawn from the figure is that in both hill cases, the deficit remains significant at long distances downstream the W/T (even greater than $40 \mathrm{D}$ ). The decay rate is slower for the quasi-3D hill. On the contrary, in the flat terrain case, the deficit has

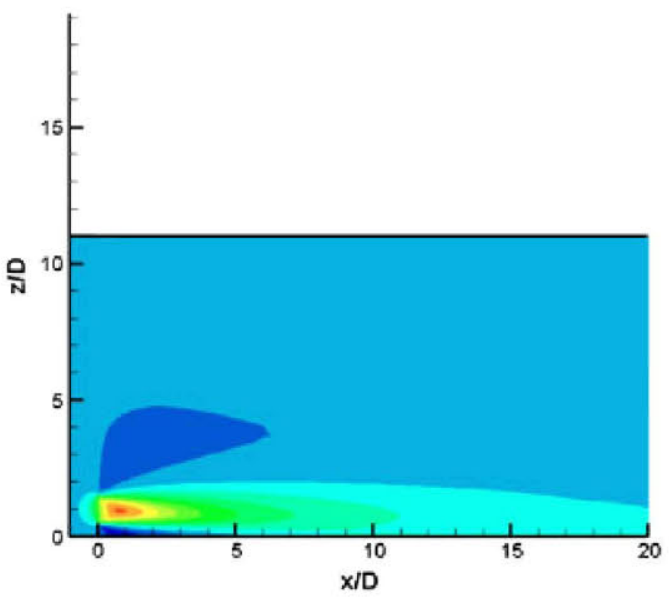

(a)

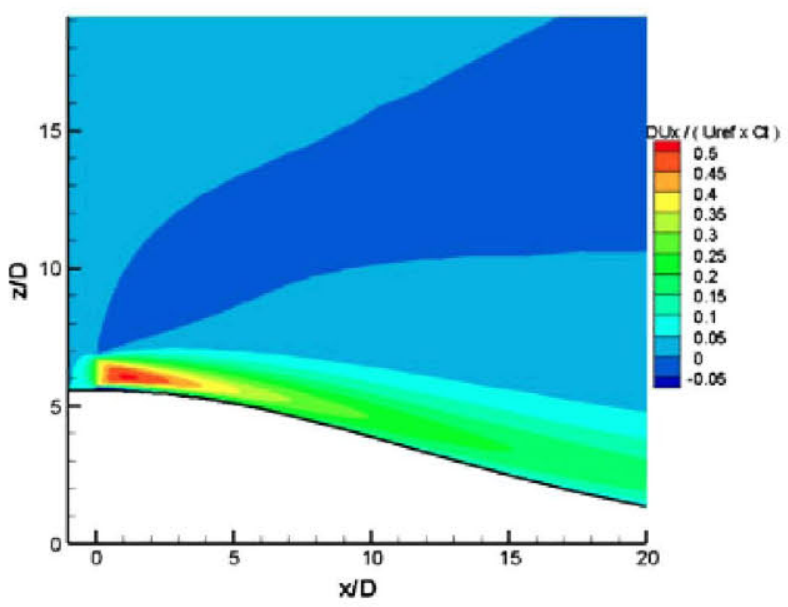

(b)

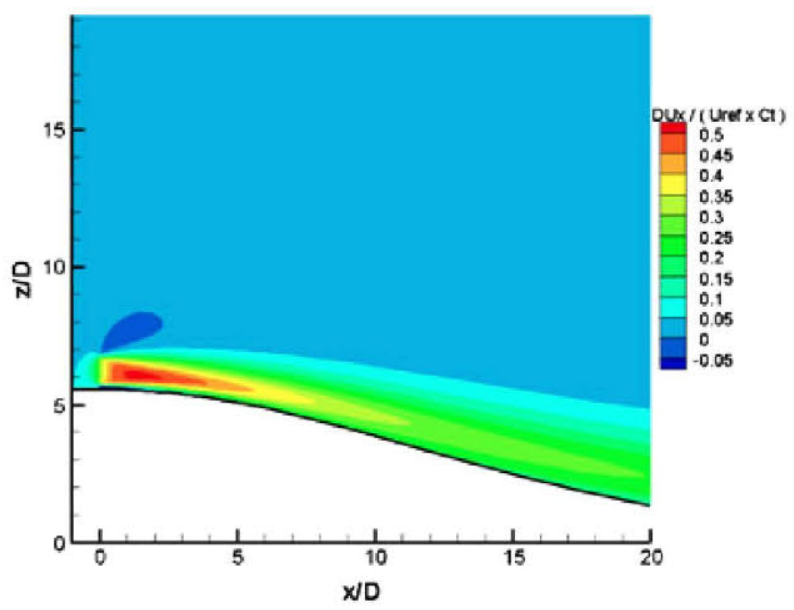

(c)

Figure 12. Velocity deficit contours at plane $y=0$ for $T t_{\text {in }}=13 \%$. (a) Flat terrain, (b) axisymmetric hill and (c) quasi-3D hill. 


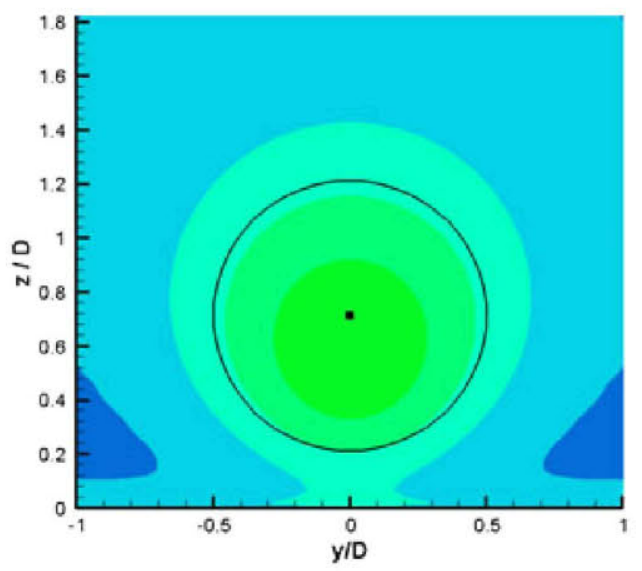

(a)

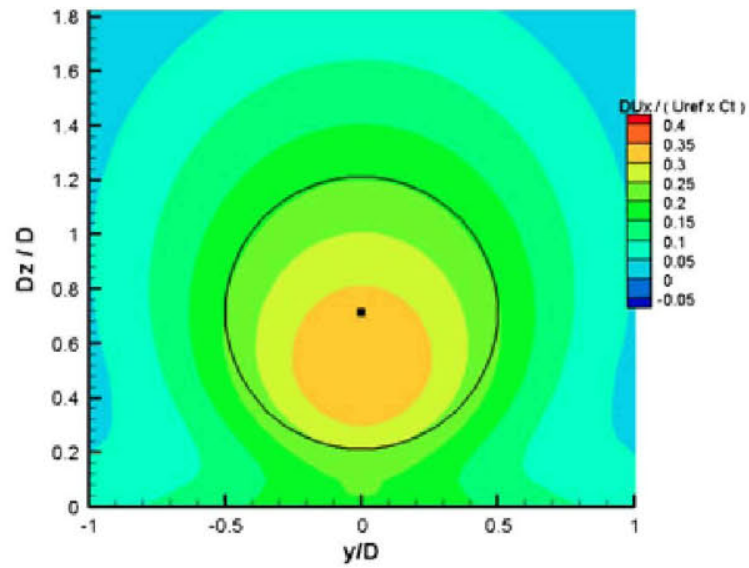

(b)

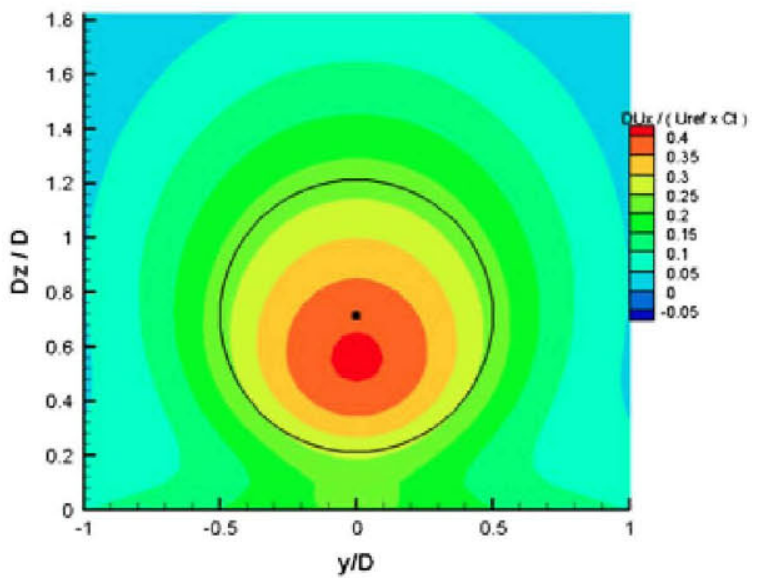

(c)

Figure 13. Velocity deficit contours $5 D$ downstream the $W / T$ for $T /$ in $=13 \%$. (a) Flat terrain, (b) axisymmetric hill and (c) quasi-3D hill.

been practically smoothed out at $20 \mathrm{D}$. The increase of the turbulence level results in a faster flow recovery at long distances as expected. However, it is noticeable that the velocity deficit at hub height is not always monotonously decreasing. This is mainly observed in the quasi-3D case and is more pronounced for the $T I_{\text {in }}=20 \%$ case. In the same figure, the predicted $C_{\mathrm{T}}$ provided by the run without $\mathrm{W} / \mathrm{T}$ is shown at the legend, and it is observed to decrease as $T I_{\text {in }}$ increases. This is a result of the fact that the increase in turbulence intensity, which is equivalent to increasing the roughness, changes the shape of the boundary layer leading to a higher velocity at hub height and subsequently to a lower $C_{\mathrm{T}}$.

The velocity deficit vertical profiles are plotted in Figure 11 at increasing distances downstream the W/T. A straightforward observation is the modification of the wake geometry with turbulence, especially in the $T I_{\text {in }}=20 \%$ case, which is responsible for the aforementioned non-monotonous variation of the axial deficit. The height of the maximum velocity deficit reduces with increasing $T I_{\text {in }}$ and for $T I_{\text {in }}=20 \%$ is located close to the ground. Another remark is that the predicted velocity deficit keeps higher values in the quasi-3D hill terrain, denoting a slower decay rate which was also seen in Figure 10 .

A visual representation of the deficit features is made using contour plots shown in Figures 12 and 13 . The deficit contours at the plane $y=0$ are compared in the first figure for the two hills and the flat terrain case. The wake evolution at long distances in both hill cases, and particularly in the quasi-3D case, contrasts to the rapid recovery in the flat terrain case. A detailed illustration of the wake geometry can be made by focusing on the region behind the W/T at a plane parallel to the rotor disk. In Figure 13, the deficit contours are presented at $5 \mathrm{D}$ downstream the W/T for $T I_{\text {in }}=13 \%$. In the flat terrain case, the wake center is about $0.1 \mathrm{D}$ lower than hub height at $5 \mathrm{D}$ downstream. In the axisymmetric and the quasi-3D hill cases, the height difference between wake center and hub becomes about $0.2 \mathrm{D}$ at $5 \mathrm{D}$ downstream. 
Another important observation is the effect of the terrain slope on the velocity deficit. A change in $L$ in Equation (12) from 1750 to $3500 \mathrm{~m}$ results in smoothening the terrain by reducing the mean slope of the hill from 0.4 to 0.2 . In Figure 14, the streamwise variation of deficit shows that the decay rate of the velocity deficit significantly depends on the terrain slope. By reducing the mean slope of the hill to half the decay rate is almost doubled. This effect is similar for both axisymmetric and quasi-3D hills and is confirmed in the representation of the vertical profiles of the velocity deficit. It becomes significant for distances greater than $5 \mathrm{D}$ (see Figure 15). In the same figure, it is depicted that the modification in the terrain slope also affects the shape of the wake geometry. Finally, the drastic effect of the wind direction on the decay rate of deficit should be underlined. As depicted in Figure 16, a change in the wind direction from 0 to $30^{\circ}$ significantly increases the decay rate. At $30^{\circ}$ wind direction, the decay rate of deficit is comparable to that of flat terrain. This effect of the wind direction on the deficit is confirmed by the profiles of Figure 17. The height of the maximum deficit remains constant; its level however attenuates fast as the wind direction changes from 0 to $30^{\circ}$. For the $30^{\circ}$ case, the velocity deficit is practically negligible after $20 \mathrm{D}$.

\section{CONCLUSIONS}

The work presented in this paper is part of the research being undertaken within the UpWind Integrated Project that aims to develop the design tools for next generation of large W/Ts. More specifically, the performance of wind farm and wake
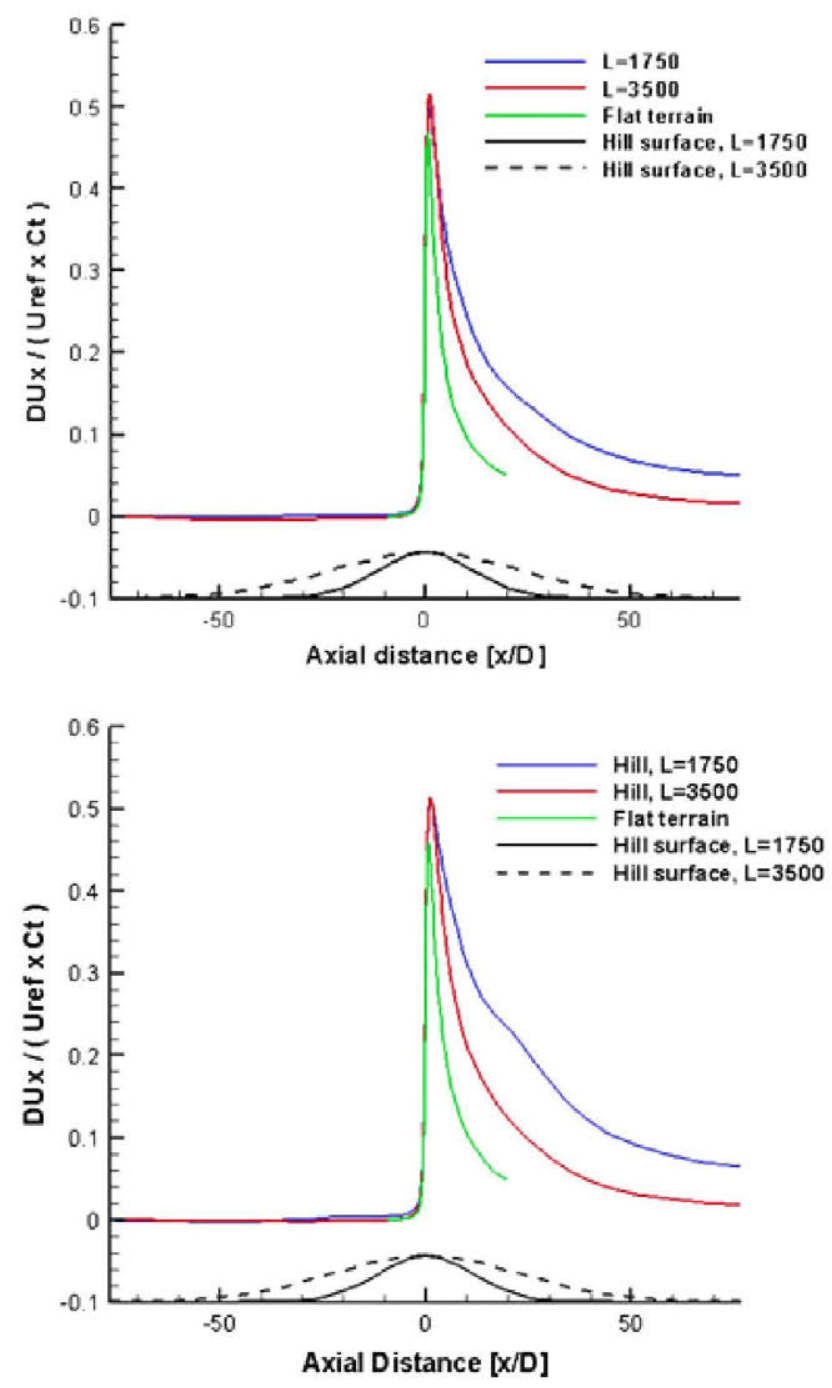

Figure 14. Velocity deficit at hub height above the Gaussian hill, for different mean hill slopes. Upper: axisymmetric hill. Lower: quasi-3D hill. 

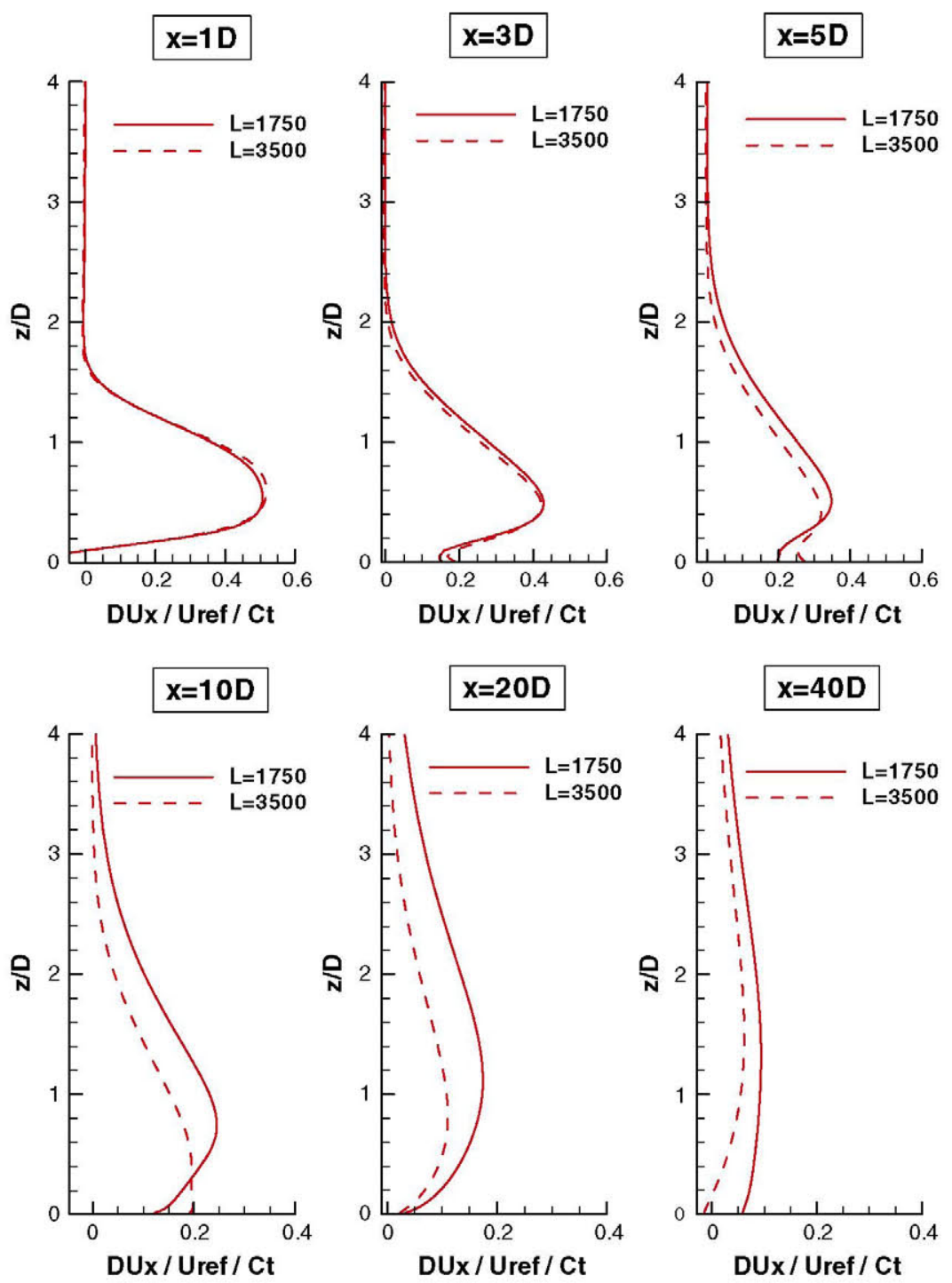

Figure 15. Vertical profiles of velocity deficit at $1,3,5,10,20$ and $40 \mathrm{D}$ downstream the W/T for different mean slopes of the axisymmetric hill. $L=1750$ corresponds to a mean slope of 0.4 , and $L=3500$ corresponds to a mean slope of 0.2 .

models is being examined in a complex terrain environment. The obvious breakthrough in this kind of application stems from the fact that is the first time that CFD tools are employed for power prediction in large wind farms in complex terrain. The challenges associated in the application of the CFD tools in complex terrain, where there are few pre-existing measurements of ambient wind and turbulence, and even fewer studies of power estimations, spring from the fact that despite the improvement of the single wake predictions with CFD tools in flat terrain, there is still space for improvements toward the diminishment of the related uncertainties in complex terrain. In complex terrain, the topography effects introduce extra sources of uncertainty in the modeling, both of the flow and the W/T, as discussed in the present paper. Apart from the need for establishing turbulence models calibrated for atmospheric flow conditions, the modeling of the wake effects in complex terrain becomes ambiguous since there is no obvious reference wind speed for the application of the actuator disk theory.

Two CFD Navier-Stokes solvers, CRES-flowNS and CFDWake, were used to estimate the performance of a large wind farm located in complex terrain. The two codes have been developed at two different institutions, the main difference being that CRES-flowNS is developed in-house, whereas CFDWake is based on the well-known FLUENT package. Besides this, CRES-flowNS uses the standard $k-\omega$ turbulence model with a correction for the near-wake turbulence, whereas CFDWake uses the standard $k-\varepsilon$ model. The constants of both turbulence models have been modified for neutral atmospheric conditions. To reduce the uncertainty regarding the comparison of the predictions by the two solvers, the computational domain 


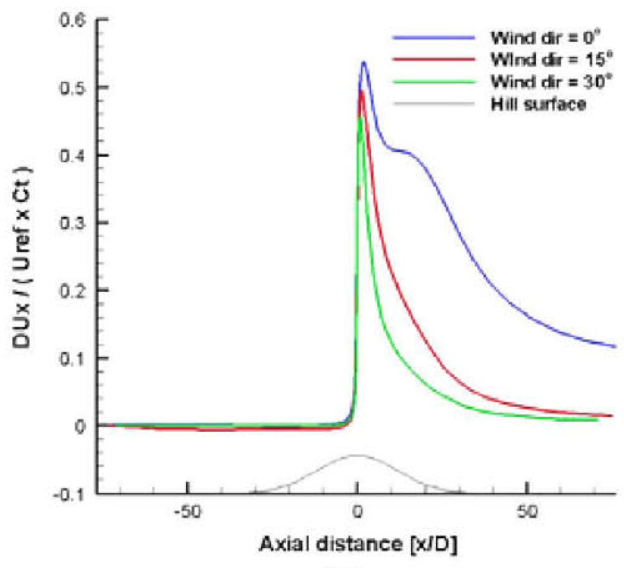

(a)

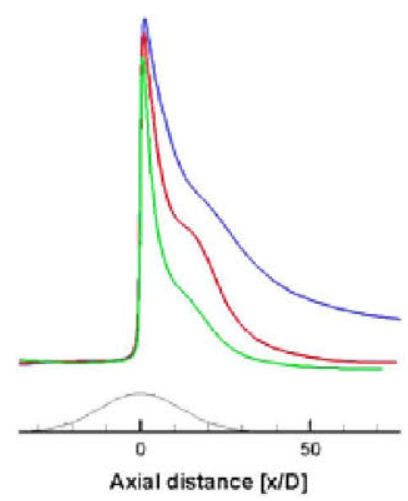

(b)

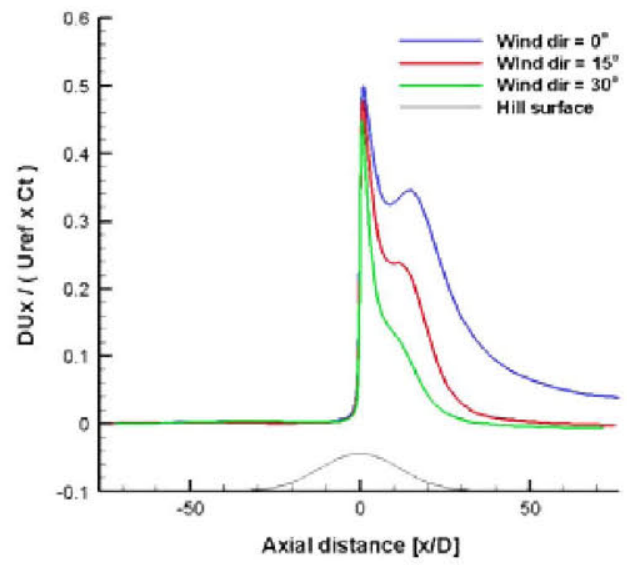

(c)

Figure 16. Velocity deficit at hub height above the quasi-3D hill, for various wind directions. (a) $T I_{\text {in }}=5 \%$, (b) $T I_{\text {In }}=13 \%$ and (c) $T$ I $_{\text {in }}=20 \%$.
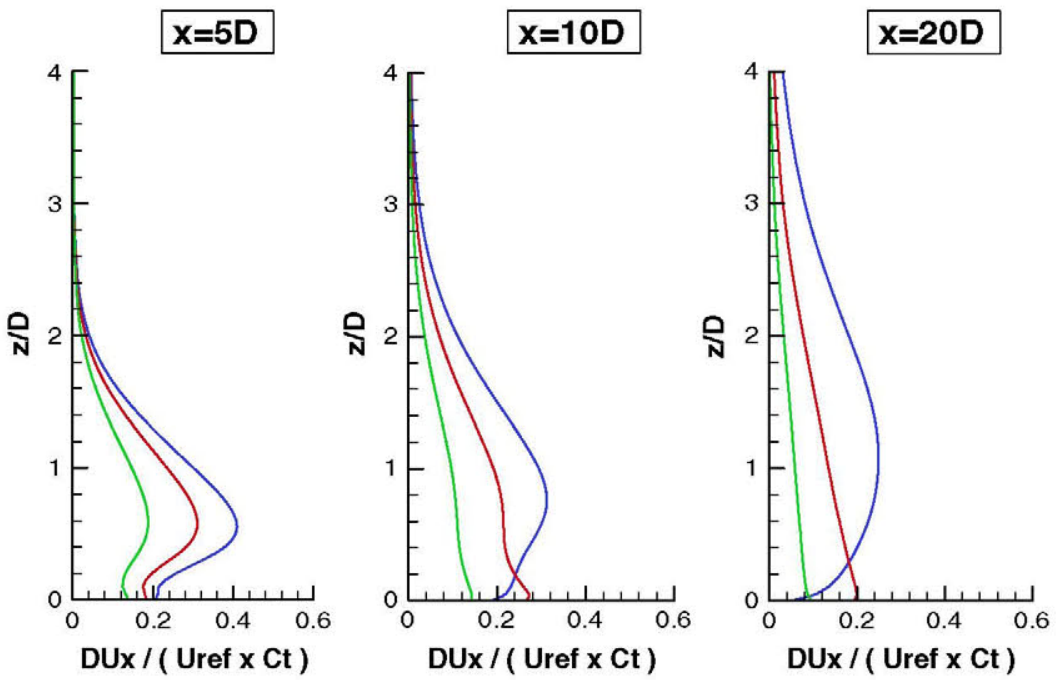

Figure 17. Vertical profiles of velocity deficit at 5, 10 and $20 \mathrm{D}$ downstream the $\mathrm{W} / \mathrm{T}$ for various wind directions of quasi-3D hill. Blue line, $0^{\circ}$; red line, $15^{\circ}$; green line, $30^{\circ}$. 
and the mesh were similar. The predictions of the streamwise wind speed for the cases without the presence of W/Ts confirmed that the two CFD solvers produce similar results, with some small differences attributed to the different turbulence models and the terrain discretization.

However, significant differences arose when the W/T modeling was introduced, although both solvers utilized the actuator disk concept, mainly, because of the different ways of defining the reference wind speed required for the thrust estimation and, secondly, of the effect of the near-wake turbulence correction used in the CRES-flowNS solver. CFDWake adopted the exact definition of the reference wind speed by performing sequential runs to calculate the wind speeds at the locations of the W/Ts, taking into account only the effect of the upstream rows of W/Ts. Predictions were satisfactory; however, this technique is possible only when the wind direction is nearly perpendicular to the W/T rows. On the other hand, CRES-flowNS applied two different approximations for the estimation of the reference wind speed that require a single computation for the entire wind farm: the wind speed value $1 \mathrm{D}$ upstream of the rotor disk at hub height is used as reference in the first, whereas an induction factor-based wind speed is used as reference in the second. For the first method to be effectively applied in complex terrain, a correction of the estimated reference wind speed $1 \mathrm{D}$ upstream of the rotor to account for the ellipticity of the flow is required. As a first approximation, this correction can be obtained from simulations in flat terrain for different thrust coefficients. The second method allows the determination of the reference wind speed without considering any distance from the turbine; however, its potential and uniform flow approximations seem to degrade its performance in complex terrain.

Predictions by WAsP for the same wind farm were also used for comparisons. However, they tend to have less variability than the observed power or the predictions from the CFD models but broadly reproduce the power variations along the turbine rows, which are related to the terrain effects.

The second issue investigated in the present paper was the effect of the terrain on wake development and its correlation with the wake development in flat terrain. To this end, a numerical study was carried out simulating the flow around an axisymmetric and a quasi-3D Gaussian hill, with and without a W/T on the top of the hill. It was found that the presence of the hill affects the development of the wake in a complicated way. In general, the velocity deficit and the wake geometry cannot be predicted by a linear superposition of the 'terrain' and the 'wake' effect. In a hilly terrain, the velocity deficit remained significant for much longer distances than in a flat terrain. A smoother terrain resulted in a higher deficit decay rate. In the simple case of a Gaussian hill, it seemed that there was an analogy between the mean slope of the hill and the predicted velocity deficit at hub height. An increase in the turbulence intensity caused a faster flow recovery and modified the wake geometry leading in some cases to a non-monotonously decrease of the velocity deficit at hub height with distance. The wake center moved to lower heights in comparison with the flat terrain, suggesting a modification of the wake geometry. Finally, a change in the wind direction affected drastically the velocity deficit in the wake region.

\section{ACKNOWLEDGEMENTS}

This work was partly funded by the European Commission and by the Greek Secretariat for Research and Technology under contract SES6 019945 (UpWind Integrated Project). The relevant wind farm owner is acknowledged for supplying the project with the data for the model evaluation.

\section{REFERENCES}

1. Ainslie JF. Calculating the flow field in the wake of wind turbines. Journal of Wind Engineering and Industrial Aerodynamics 1988; 27: 213-224.

2. Katic I, Højstrup J, Jensen NO. A simple model for cluster efficiency. Proceedings of the European Wind Energy Association, Rome, 1986.

3. Jensen NO. A note on wind generator interaction. Technical Report M-2411, Ris $\varnothing$ National Laboratory, Roskilde, Denmark, 1983.

4. Crespo A, Hernandez J, Fraga E, Andreu C. Experimental validation of the UPM computer code to calculate wind turbine wakes and comparison with other models. Joumal of Wind Engineering and Industrial Aerodynamics 1988; 27: 77-88.

5. Landberg L, Myllerup L, Rathmann O, Petersen EL, Jørgensen BH, Badger J, Mortensen NG. Wind resource estimation-an overview. Wind Energy 2003; 6: 261-271.

6. Mortensen NG, Heathfield DN, Myllerup L, Landberg L, Rathmann O. Getting started with WAsP 9. Technical Report Risø-I-2571(E), Risø National Laboratory, Roskilde, Denmark, 2007.

7. Walmsley JL, Troen I, Lalas DP, Mason PJ. Surface-layer flow in complex terrain: comparison of models and full-scale observations. Boundary-Layer Meteorology 1990; 52: 259-281. 
8. Cattin R, Schaffner B, Kunz S. Validation of CFD wind resource modeling in highly complex terrain, Proceedings of the 2006 European Wind Energy Conference and Exhibition, Athens, Greece, 2006.

9. Cabezón D, Sanz J, Beeck JV. Sensitivity analysis on turbulence models for the ABL in complex terrain, 2007 European Wind Energy Conference and Exhibition, Milan, Italy, 2007.

10. Politis ES, Chaviaropoulos PK. Micrositing and Classification of Wind Turbines in Complex Terrain. Proceedings of the 2008 European Wind Energy Conference \& Exhibition, Brussels, 31 March-3 April 2008; 126-130.

11. Palma JMLM, Castro FA, Ribeiro LF, Rodrigues AH, Pinto AP. Linear and nonlinear models in wind resource assessment and wind turbine micro-siting in complex terrain. Journal of Wind Engineering and Industrial Aerodynamics 2008; 96: 2308-2326.

12. Bechmann A, Sørensen NN. Hybrid RANS/LES method for wind flow over complex terrain. Wind Energy 2010; 13: 36-50.

13. Sørensen JN, Shen WZ. Numerical modelling of wind turbines. Journal of Fluids Engineering 2002; 124: $393-399$.

14. Frandsen S, Barthelmie R, Pryor S, Rathmann O, Larsen S, Højstrup J. Analytical modelling of wind speed deficit in large offshore wind farms. Wind Energy 2006; 9: 39-53.

15. Barthelmie RJ, Hansen K, Frandsen ST, Rathmann O, Schepers JG, Schlez W, Phillips J, Rados K, Zervos A, Politis ES, Chaviaropoulos PK. Modelling and measuring flow and wind turbine wakes in large wind farms offshore. Wind Energy 2009; 12: 431-444.

16. Chaviaropoulos PK, Douvikas DI. Mean-flow-field simulations over complex terrain using a 3D Reynolds Averaged Navier-Stokes solver. Proceedings of ECCOMAS, Vol. 1, Part 2, 1998; 842-848.

17. Prospathopoulos JM, Politis ES, Chaviaropoulos PK. Modelling wind turbines in complex terrain, Proceedings of the 2008 European Wind Energy Conference \& Exhibition, Brussels, 2008.

18. El Kasmi A, Masson C. An extended $k-\varepsilon$ model for turbulent flow through horizontal axis wind turbines. Journal of Wind Engineering and Industrial Aerodynamics 2008; 96: 103-122.

19. Réthoré P-E, Sorensen NN, Bechman A. Modeling issues with wind turbine wake and atmospheric turbulence, Proceedings of 'The Science of Making Torque from Wind' Conference, Crete, Greece, June 2010.

20. Prospathopoulos JM, Politis ES, Rados K, Chaviaropoulos PK. Evaluation of the effects of turbulence model enhancements on wind turbine wake predictions. Wind Energy 2011; 14: 285-300. DOI: 10.1002/we.419.

21. Durbin PA. On the $k-\varepsilon$ stagnation point anomaly. International Journal of Heat and Fluid Flow 1996; 17: 89-90.

22. Cabezón D, Sanz J, Martí I, Crespo A. CFD modelling of the interaction between the surface boundary layer and rotor wake. Comparison of results obtained with different turbulence models and mesh strategies, Proceedings of the 2009 European Wind Energy Conference \& Exhibition, Marseille, France, March 2009.

23. Sanz J, Cabezón D, Martí I, Patilla P, van Beeck J. Numerical CFD modelling of non-neutral atmospheric boundary layers for offshore wind resource assessment based on Monin-Obukhov theory, Proceedings of the 2008 European Wind Energy Conference \& Exhibition, Brussels, Belgium, April 2008.

24. Panofsky H, Dutton J. Atmospheric Turbulence. Wiley: New York, 1984.

25. Eggleston DM, Stoddard FS. Wind Turbine Engineering Design. Van Nostrand Reinhold: New York, 1987; 30-35, 58.

26. Dekker JWM, Pierik JTG. (eds) European Wind Turbine Standards II, ECN-C-99-073, 1999.

27. Troen I, Petersen EL. European Wind Atlas. Risø National Laboratory: Roskilde, Denmark, 1989.

28. Mortensen NG, Bowen AJ, Antoniou I. Improving WAsP predictions in (too) complex terrain, Proceedings (online) of the 2006 European Wind Energy Conference and Exhibition, Athens, Greece, 27 February-2 March 2006. 